\title{
AoI-Minimal Trajectory Planning and Data Collection in UAV-Assisted Wireless Powered IoT Networks
}

\author{
Huimin $\mathrm{Hu}$, Ke Xiong, Member, IEEE, Gang Qu, Senior Member, IEEE, Qiang Ni, Senior \\ Member, IEEE, Pingyi Fan, Senior Member, IEEE, Khaled Ben Letaief, Fellow, IEEE
}

\begin{abstract}
This paper investigates the UAV-assisted wireless powered IoT system, where a UAV takes off from a data center, flies to each of the ground sensor nodes (SNs) in order to transfer energy and collect data form the SNs, and then returns to the data center. For such a system, an optimization problem is formulated to minimize the average age of information (AoI) of the data collected from all ground SNs. Since the average AoI depends on the UAV's trajectory, the time required for energy harvesting (EH) and data collection for each $\mathrm{SN}$, these factors need to be optimized jointly. Moreover, instead of the traditional linear EH model, we employ a non-linear model because the behavior of the EH circuits are non-linear by nature. To solve this non-convex problem, we propose to decompose it into two sub-problems, i.e., a joint energy transfer and data collection time allocation problem and a UAV's trajectory planning problem. For the first sub-problem, we prove that it is convex and give an optimal solution by using Karush-Kuhn-Tucker (KKT) conditions. This solution is used as the input for the second sub-problem, and we solve optimally it by designing dynamic programming (DP) and ant colony (AC) heuristic algorithms. Simulation results show that the DP-based algorithm obtains the minimal average AoI of the system, and the AC-based heuristic finds solutions with nearoptimal average AoI. The results also reveal that the average AoI increases as the flying altitude of the UAV increases and linearly with the size of the collected data at each ground SN.
\end{abstract}

Index Terms-Age of Information, energy harvesting, Internetof-things (IoT), time allocation, trajectory design, UAV-assisted networks.

This paper is partly supported by the Fundamental Research Funds for the Central Universities (No. 2019YJS035), by National Natural Science Foundation of China (NSFC) under Grant no. 61671051, no. 61725101, no. 61961130391 and no. U1834210, by the Royal Society Newton Advanced Fellowship under Grant no. NA191006, and also by the State Key Lab of Rail Traffic Control and Safety under Grant RCS2020ZT010. (Corresponding author: Ke Xiong.)

$\mathrm{H}$. Hu and K. Xiong are with the School of Computer and Information Technology, Beijing Jiaotong University, Beijing 100044, China, with the Beijing Key Laboratory of Traffic Data Analysis and Mining, Beijing Jiaotong University, Beijing 100044, China, and also with the Beijing Key Laboratory of Security and Privacy in Intelligent Transportation, Beijing Jiaotong University, Beijing 100044, China (e-mail: kxiong@bjtu.edu.cn).

G. Qu is with the Department of Electrical and Computer Engineering, University of Maryland at College Park, College Park, MD 20742 USA (email: Gangqu@umd.edu).

Q. Ni is with the School of Computing and Communications and Data Science Institute, Lancaster University, England (e-mail: q.ni@lancaster.ac.uk).

P. Fan is with the Department of Electronic Engineering and the Beijing National Research Center for Information Science and Technology, Tsinghua University, Beijing, P. R. China. (email: fpy@ @singhua.edu.cn).

K. B. Letaief is with the School of Engineering, Hong Kong University of Science and Technology, Hong Kong, and also with the Peng Cheng Laboratory, Shenzhen, China. (e-mail: eekhaled@ece.ust.hk).

\section{INTRODUCTION}

\section{A. Background}

With the development of Internet of Things (IoT), various real-time status information updating applications, such as intelligent transportation [1], environmental monitoring [2], safety protection and health monitoring [3], are spawned. In such applications, the generated status information is required to be delivered to the destination as quickly as possible for online data analyzing and decision-making. The outdated information may result in erroneous control, even causing big disasters. Therefore, to guarantee the freshness of the received data at the destination in status information updating systems is extremely essential.

In order to characterize the freshness of information, a new performance metric, i.e., age of information (AoI), was proposed in [4]. AoI depicts the elapsed time since the generation of the latest received update, which is able to capture the freshness of the information from the perspective of the destination [4]. Thus, it has attracted increasing attention [4]-[14]. Among existing works, some ones focused on the AoI of different queuing systems [4]-[6], some focused on the AoI-based scheduling of update packet transmissions from different source nodes [7]-[10], and some others focused on the AoI performance of energy harvesting (EH) driven wireless networks [11]-[14]. Their obtained results show that the AoIbased design is able to guarantee the information freshness of real-time status information updating systems and is much different from traditional delay-based and throughput-based network design.

On the other hand, due to the mobility and flexibility, unmanned aerial vehicle (UAV) widely is regarded as a promising approach to aid outdoor status information updating systems in collecting data (CD) [15], which has been considered as a candidate technology for future 6G networks and has wide applications in various scenarios. For instance, in smart agriculture systems and smart meadow systems, sensor nodes (SNs) are deployed to monitor a variety of environmental parameters and animals' and plants' status, where the sampled data can be collected and carried to the data center by UAV for analyzing and intelligent decision-making, see e.g., [16], [17]. In [16], a novel data acquisition framework was proposed to increase the efficiency of the data gathering, where the UAV was used as a relay to collect the sensed data from the SNs. In [17], the SNs' mechanism selection was studied to enhance 
reliable and energy-efficient data collection, where the UAV was used as a mobile alarm clock to wake up the SNs.

It is a fact that by equipping with communication transceivers, UAVs can be used as aerial relays or mobile base stations to help improve the performance of terrestrial wireless communication systems [18]-[20].

\section{B. Related Works}

Due to the significance of AoI and the popularity of UAV in IoT systems, AoI-aware UAV-aided wireless network design has attracted increasing interests [21]-[24]. In [21], the UAV's trajectory was optimized to minimize both the average AoI of the system and the maximum AoI of different wireless sensors, where the UAV was used to collect the SNs' data. In [22], the UAV's trajectory and data acquisition mode were jointly optimized to minimize average AoI of all the SNs, where the UAV was allowed to acquire the data in hovering, flying or hybrid mode. In [23], a UAV's trajectory planning strategy was proposed to minimize the maximum AoI of a UAV-enabled wireless sensor network, where the balance between the SNs' uploading time and the UAV's flight time was achieved. In [24], the UAV's flight trajectory and service time allocation were jointly optimized to minimize the overall peak AoI of the system, where the UAV was used as a mobile relay to help the information transmission between source-destination pair.

However, in aforementioned works, the $\mathrm{SN}$ or the source node was assumed to be with fixed energy supply and powered by small-size batteries, which are with limited capacity and require to be replaced or recharged periodically. In rigorous environments including poisonous and dangerous areas, it may be highly inefficient or even impractical to manually replace or recharge batteries of the distributed SNs. Thereby, EH technologies were introduced into UAV-assisted IoT systems to power low-power devices [25]. Among different EH technologies, the frequency signal (RF)-based EH is artificially controllable and able to provide stable power supply [26], [27], so it is considered as a promising solution to power future IoT and low-power wireless sensor networks [28]-[31]. Although UAVs are self-energy-limited, the energy required by communication and RF energy transfer at the UAV is much smaller than that required by its flying and hovering. Therefore, UAVs are also used as mobile RF energy sources for ground sensors in many recent works, see e.g., [32][34]. As a result, some recent works began to study UAVaided wireless network with RF-based EH [35], [36]. In [35], outage probability was derived for different urban environment parameters, where the UAV acted as a relay to harvest energy and collect data and then to forward data. In [36], the average throughput was maximized subject to the energy causality constraint with a generalized harvest-transmit-store model.

\section{Motivations and Contributions}

Nevertheless, the purpose of aforementioned works on UAVaided wireless network with RF-based EH, see e.g., [35], [36] was to analyze the outage performance or maximize system throughput. To the best of our knowledge, little attention has been devoted to the AoI-based UAV-assisted wireless network with RF-based EH.

To fill this gap, we investigate the joint optimization of trajectory and time assignment for AoI-based UAV-assisted wireless powered IoT system, where a UAV takes off from a data center, flies to each SN in turn to transfer energy, then collects data from the $\mathrm{SN}$, and finally returns to the data center. Different from existing works, see e.g., [35], [36], our goal is to minimize the average AoI of the system. Moreover, our considered model is also different from theirs. Particularly, in [35], the UAV was assumed to be with very limited energy and charged by ground base stations, but in our work, the UAV acts as a mobile energy source and is used to charge ground lowpower IoT devices. Although, in [36], the UAV served as an energy source, it was not used for data collection. Differently, in our work, the UAV is used as not only an energy source but also a mobile data collector. Besides, in [35] and [36], the traditional ideal linear EH model was adopted, but in our work, the non-linear EH model [37] presented based on real data measurement is employed for system design. The main contributions of this paper are summarized as follows.

- An optimization problem is formulated to minimize the average AoI of the data collected from all ground SNs by jointly optimizing UAV's trajectory, the time required for energy harvesting and the time required for data collection at each SN.

- Since the problem is non-convex and has no known solution, a solution framework is presented to solve it, where the primary problem is first decomposed into two sub-problems, i.e., a joint energy transfer and data collection time allocation problem and a UAV's trajectory planning problem. It is proved that such a decomposition does not loss the optimal solution to primary problem. For the first subproblem, it is convex and solved by using Karush-Kuhn-Tucker (KKT) conditions. For the second subproblem, based on the obtained optimal solution to the first subproblem, two different algorithms are designed to solve it. Particularly, a dynamic programming (DP)-based algorithm is designed to find the global optimal solution by checking all candidate solutions, which may be too complex when the number of SNs are large. Therefore, as an alternative, an ant colony (AC)-based algorithm is designed to find a suboptimal solution with low complexity by employing a positive feedback mechanism to make the iteration converge and finally approaches the optimal solution.

- Simulation results are provided to show the performance of the presented algorithms. It shows that the DP-based algorithm obtains the minimal average AoI of the system, and the AC-based algorithm finds the near-optimal average AoI. Moreover, compared with traditional linear EH model, employing the non-linear EH model decreases the average AoI as real systems are operated based on the $\mathrm{EH}$ circuits with non-linear features. It is also observed that as the flying altitude of the UAV increases, the average AoI increases, and the gap between the average AoI achieved by traditional linear EH model and the non-linear EH 


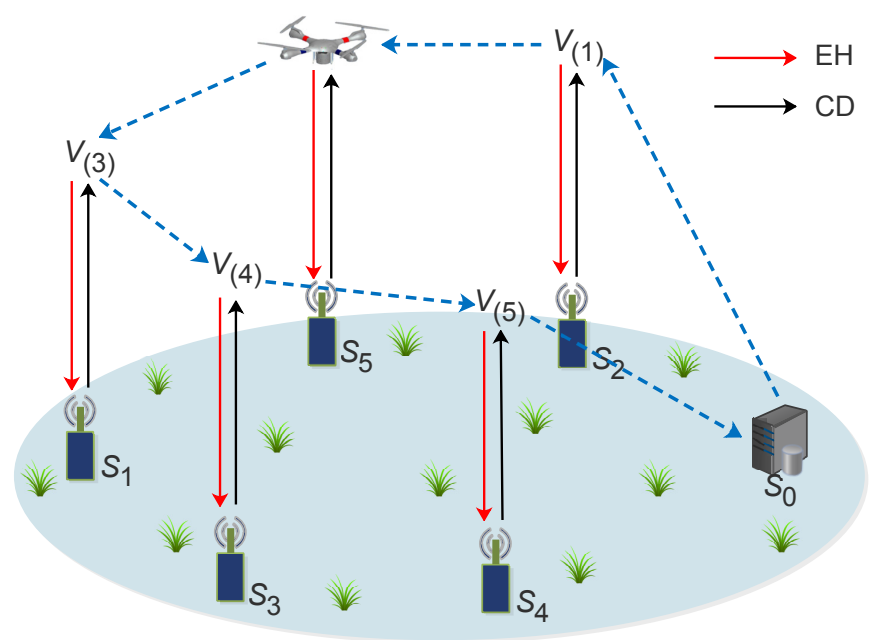

Fig. 1. An illustrative model of UAV-assisted IoT networks: UAV flies in a trajectory $S_{0} \rightarrow V_{(1)} \rightarrow V_{(2)} \rightarrow V_{(3)} \rightarrow V_{(4)} \rightarrow V_{(5)} \rightarrow S_{0}$ to transmit energy and then collect the latest sensing information form each user $S_{i}$ $(i=1, \ldots, 5)$, and flies back to the data center $S_{0}$.

model is also enlarged.

The rest of this paper is organized as follows. In Section II, we describe the system model for UVA-assisted energy transfer and data collection, and formulate the average AoI minimization problem. In Section III, we present our proposed solution framework and the specific solution steps of the two presented algorithms. In Section IV, we present some simulation results to discuss the system performance. Finally, this paper is concluded in Section V.

\section{System Model AND PROBlem Formulation}

\section{A. System Model}

As shown in Fig. 1, we consider a UAV-assisted wireless powered IoT system that consists of one UAV equipped with a half-duplex hybrid access point (HAP), a data center $S_{0}$, and $M$ ground sensor nodes (SNs) denoted by $\mathbf{S}=$ $\left\{S_{1}, S_{2}, \ldots, S_{M}\right\} . M$ ground SNs are randomly distributed in a large area to monitor a variety of physical processes, and each node $S_{i} \in\left\{S_{0}\right\} \cup \mathbf{S}$ with $i \in\{0,1,2, \cdots, M\}$ is located at the position of $s_{i}=\left(x_{i}, y_{i}\right)$. The UAV performs wireless energy transfer to power the SNs and also collects data from them. Specifically, the UAV takes off from the data center $S_{0}$, and flies to the SN and transfers energy to it. Then the SN uses the harvested energy to upload its data to the UAV. The UAV completes the energy transfer and data collection tasks for the $M$ SNs one by one according to a predetermined flight trajectory, i.e., $S_{0} \rightarrow V_{(1)} \rightarrow V_{(2)} \rightarrow \ldots \ldots \rightarrow$ $V_{(M)} \rightarrow S_{0}$, where $V_{(i)}$ is the $i$-th SN on flight trajectory and $\left\{V_{(1)}, V_{(2)}, \cdots, V_{(M)}\right\}$ is a permutation of $\left\{S_{1}, S_{2}, \cdots, S_{M}\right\}$. We assume that the UAV flies at a fixed altitude $H$ with a constant speed denoted by $v$. The horizontal distance between $S_{i}$ and $S_{j}$ is denoted with $d_{i, j}$, i.e., $d_{i, j}=\left\|s_{i}-s_{j}\right\|$, $\forall i, j \in\{1,2, \ldots, M\}$.

In order to charge the SN with sufficient power, the UAV needs to hover over the $\mathrm{SN}$ for some time. We denote the hovering for $\mathrm{EH}$ time as $t_{i}^{\mathrm{eh}}$ and that for UAV to collect data time as $t_{i}^{\text {cd }}, i \in\{1,2, \cdots, M\}$. The downlink power gain from the UAV to $S_{i}$ and the uplink power gain from $S_{i}$ to the UAV are denoted by $\left|h_{i}\right|^{2}$ and $\left|g_{i}\right|^{2}$, respectively. Since the channel between UAV and SNs is dominated by the line-of-sight (LoS) communication link, we have that $\left|h_{i}\right|^{2}=\left|g_{i}\right|^{2}=\frac{\kappa_{0}}{H^{\kappa}}$, where $\kappa_{0}$ denotes the reference signal power gain at the distance of one meter from the $\mathrm{SN}$, and $\kappa$ is the path loss factor [15].

\section{B. Energy Harvesting Model and Data Collection Model}

In the wireless power transfer stage, the UAV keeps transmitting RF signals to the $\mathrm{SN}$ with a fixed transmit power $P_{u}$. The power carried in the received RF signals at $S_{i}$ is

$$
P_{r}=\left|h_{i}\right|^{2} P_{u} .
$$

Based on the traditional linear EH models, the harvested power at $S_{i}$ can be described by $\Phi(P)=\eta_{i} P_{r}$, where $\eta_{i} \in$ $(0,1]$ is a constant. However, since the RF-EH circuits include various non-linearities, such as the diode or diode-connected transistor, the non-linear EH model presented in [37], [40] is adopted in this paper to characterize the non-linear behavior of the EH circuits and make our design closer to practice. With the non-linear EH model [41], the harvested power at $S_{i}$ is described by

$$
\Phi\left(P_{r}\right)=\frac{P_{\max } e^{(a b)}-P_{\max } e^{\left(-a\left(P_{r}-b\right)\right)}}{e^{(a b)}\left(1+e^{\left(-a\left(P_{r}-b\right)\right)}\right)},
$$

where $P_{\max }$ denotes the maximum output DC power, which is the saturation limitation of the EH circuits. $a$ and $b$ are constants representing some properties of the EH system such as the resistance, the capacitance and the circuit sensitivity. As a result, the energy harvested at $S_{i}$ from the UAV during the time interval $t_{i}^{\text {eh }}$ is

$$
E_{i}=\Phi\left(P_{r}\right) t_{i}^{\mathrm{eh}} .
$$

In the data collection stage, $S_{i}$ transmits its data to the UAV with the harvested energy. Thus, the uploading data rate of $S_{i}$ is expressed by

$$
R_{i}=W \log _{2}\left(1+\frac{\left|h_{i}\right|^{2} E_{i}}{t_{i}^{\mathrm{cd}} \sigma_{w}^{2}}\right),
$$

where the harvested energy during $t_{i}^{\text {eh }}$ is allocated uniformly to the data uploading over $t_{i}^{\mathrm{cd}}$, and $W$ is the system bandwidth. $\sigma_{w}^{2}$ is the noise power at the UAV. Assume that the size of data generated by $S_{i}$ is $D_{i}$. To make sure $S_{i}$ successfully uploads its data to the UAV within $t_{i}^{\text {cd }}$, the following inequality needs to be satisfied

$$
t_{i}^{\mathrm{cd}} W \log _{2}\left(1+\frac{\left|h_{i}\right|^{2} E_{i}}{t_{i}^{\mathrm{cd}} \sigma_{w}^{2}}\right) \geq D_{i} .
$$

Substituting (3) into (4), we have that

$$
t_{i}^{\mathrm{cd}} W \log _{2}\left(1+\frac{\left|h_{i}\right|^{2} \Phi\left(P_{r}\right) t_{i}^{\mathrm{eh}}}{t_{i}^{\mathrm{cd}} \sigma_{w}^{2}}\right) \geq D_{i} .
$$




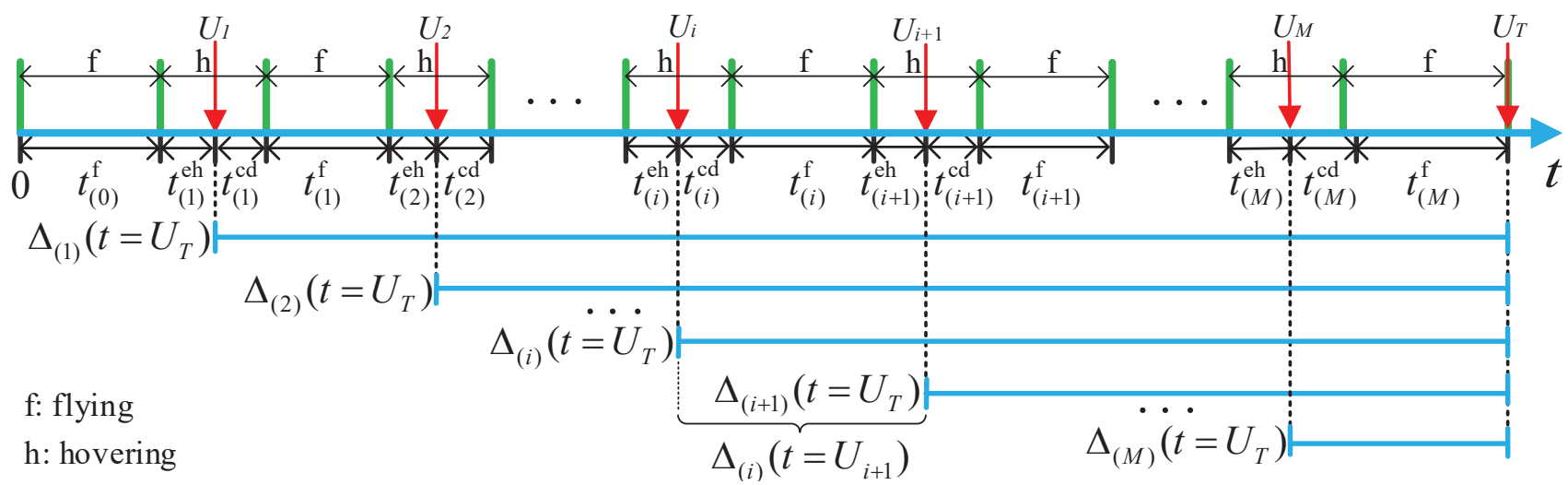

Fig. 2. The time sequence including the hovering and flying periods.

\section{AoI Models}

Let $\Delta_{(i)}(t)$ be the AoI of the data collected from $i$-th $V_{(i)}$ at time $t$. According to the definition of AoI [4], we have that

$$
\Delta_{(i)}(t)=\left(t-U_{i}\right)^{+},
$$

where $(a)^{+}=\max \{0, a\}$, and $U_{i}$ is the timestamp of starting the data collection from $i$-th $V_{(i)}$. In fact, when $t<U_{i}$, SN $V_{(i)}$ has not been visited at time $t$, we define $\Delta_{(i)}(t)=0$. For clarity, the working time framework of UAV is illustrated in Fig. 2. It can be seen that at time $U_{i+1}$, the AoI of the data collected from $V_{(i)}$ is given by

$$
\Delta_{(i)}\left(t=U_{i+1}\right)=t_{(i)}^{\mathrm{cd}}+t_{(i)}^{\mathrm{f}}+t_{(i+1)}^{\mathrm{eh}}, i=1,2, \cdots, M-1,
$$

which is composed of three parts, where $t_{(i)}^{\mathrm{f}}$ is the flying time of the UAV from $V_{(i)}$ to $V_{(i+1)}$, which is given by $t_{(i)}^{\mathrm{f}}=$ $d_{(i),(i+1)} v^{-1}$.

Let $U_{T}$ be the observation time at which all data has been carried to the data center. When the UAV returns to the data center $S_{0}$ after gathering data from all the SNs, the AoI of the data collected from $V_{(i)}$ can be given by

$$
\begin{aligned}
\Delta_{(i)}\left(t=U_{T}\right)= & \Delta_{(i)}\left(t=U_{i+1}\right)+\Delta_{(i+1)}\left(t=U_{T}\right) \\
= & \Delta_{(i)}\left(t=U_{i+1}\right)+\Delta_{(i+1)}\left(t=U_{i+2}\right) \\
& +\Delta_{(i+2)}\left(t=U_{T}\right) \\
= & \Delta_{(i)}\left(t=U_{i+1}\right)+\Delta_{(i+1)}\left(t=U_{i+2}\right) \\
& +\cdots+\Delta_{(M-1)}\left(t=U_{M}\right)+\Delta_{(M)}\left(t=U_{T}\right) \\
= & t_{(i)}^{\mathrm{cd}}+t_{(i)}^{\mathrm{f}}+t_{(i+1)}^{\mathrm{eh}}+t_{(i+1)}^{\mathrm{cd}}+t_{(i+1)}^{\mathrm{f}}+t_{(i+2)}^{\mathrm{eh}} \\
& +\cdots+t_{(M-1)}^{\mathrm{cd}}+t_{(M-1)}^{\mathrm{f}}+t_{(M)}^{\mathrm{eh}} \\
& +t_{(M)}^{\mathrm{cd}}+t_{(M)}^{\mathrm{f}} \\
& \sum_{k=i}^{M-1}\left(t_{(k)}^{\mathrm{cd}}+t_{(k)}^{\mathrm{f}}+t_{(k+1)}^{\mathrm{eh}}\right)+\left(t_{(M)}^{\mathrm{cd}}+t_{(M)}^{\mathrm{f}}\right) \\
= & \sum_{k=i}^{M}\left(t_{(k)}^{\mathrm{eh}}+t_{(k)}^{\mathrm{cd}}+t_{(k)}^{\mathrm{f}}\right)-t_{(i)}^{\mathrm{eh}},
\end{aligned}
$$

where $i=1,2, \cdots, M$.

The last term $\left\{-t_{(i)}^{\mathrm{eh}}\right\}$ in (8) indicates that $t_{(i)}^{\mathrm{eh}}$ does not contribute to AoI of $V_{(i)}$, because $V_{(i)}$ harvests energy before its data generating. In terms of (8), i.e., $\Delta_{(i)}\left(t=U_{T}\right)=$ $\Delta_{(i)}\left(t=U_{i+1}\right)+\Delta_{(i+1)}\left(t=U_{T}\right)$, and Fig. 2, one can draw the following conclusion, i.e.,

$$
\begin{gathered}
\Delta_{(1)}\left(t=U_{T}\right)>\Delta_{(2)}\left(t=U_{T}\right)>\Delta_{(3)}\left(t=U_{T}\right)>\cdots \\
>\Delta_{(M-1)}\left(t=U_{T}\right)>\Delta_{(M)}\left(t=U_{T}\right),
\end{gathered}
$$

which presents that the AoI $\Delta_{(i)}\left(t=U_{T}\right)$ of data collected from the $i$-th SN has effects on $\Delta_{(1)}\left(t=U_{T}\right), \Delta_{(2)}(t=$ $\left.U_{T}\right), \cdots$, and $\Delta_{(i-1)}\left(t=U_{T}\right)$ but has no effect on $\Delta_{(i+1)}\left(t=U_{T}\right), \Delta_{(i+2)}\left(t=U_{T}\right), \cdots$, and $\Delta_{(M)}\left(t=U_{T}\right)$. The reason is that the AoI of data collected in the future is irrelevant to the AoI of data collected in the past, but it will affect the AoI of data collected in the observed time period.

The average AoI of all data collected from $M$ SNs is defined by

$$
\bar{\Delta}=\frac{1}{M} \sum_{i=1}^{M} \Delta_{(i)}\left(t=U_{T}\right) .
$$

Lemma 1. The average AoI of the considered UAV-assisted wireless powered network can be expressed as

$$
\bar{\Delta}=\sum_{i=1}^{M} \frac{i}{M}\left(t_{(i)}^{\mathrm{eh}}+t_{(i)}^{\mathrm{cd}}+t_{(i)}^{\mathrm{f}}\right)-\frac{1}{M} \sum_{i=1}^{M} t_{(i)}^{\mathrm{eh}} .
$$

Proof. Substituting (8) into (10), we then derive $\bar{\Delta}$ as

$$
\begin{aligned}
\bar{\Delta}= & \frac{1}{M}\left(\Delta_{(1)}\left(t=U_{T}\right)+\Delta_{(2)}\left(t=U_{T}\right)+\Delta_{(3)}\left(t=U_{T}\right)\right. \\
& \left.+\cdots+\Delta_{(M-1)}\left(t=U_{T}\right)+\Delta_{(M)}\left(t=U_{T}\right)\right) \\
= & \frac{1}{M}\left[\sum_{k=1}^{M}\left(t_{(k)}^{\mathrm{eh}}+t_{(k)}^{\mathrm{cd}}+t_{(k)}^{\mathrm{f}}\right)-t_{(1)}^{\mathrm{eh}}+\sum_{k=2}^{M}\left(t_{(k)}^{\mathrm{eh}}+t_{(k)}^{\mathrm{cd}}\right.\right. \\
& \left.\left.+t_{(k)}^{\mathrm{f}}\right)-t_{(2)}^{\mathrm{eh}}+\cdots+\sum_{k=M}^{M}\left(t_{(k)}^{\mathrm{eh}}+t_{(k)}^{\mathrm{cd}}+t_{(k)}^{\mathrm{f}}\right)-t_{(M)}^{\mathrm{eh}}\right] \\
= & \frac{1}{M}\left[\sum_{i=1}^{M} i\left(t_{(i)}^{\mathrm{eh}}+t_{(i)}^{\mathrm{cd}}+t_{(i)}^{\mathrm{f}}\right)-\sum_{i=1}^{M} t_{(i)}^{\mathrm{eh}}\right] \\
= & \sum_{i=1}^{M} \frac{i}{M}\left(t_{(i)}^{\mathrm{eh}}+t_{(i)}^{\mathrm{cd}}+t_{(i)}^{\mathrm{f}}\right)-\frac{1}{M} \sum_{i=1}^{M} t_{(i)}^{\mathrm{eh}} .
\end{aligned}
$$




\section{Problem Formulation}

Our goal is to optimally plan a trajectory and also allocate the time for $\mathrm{EH}$ and data collection for all SNs in order to minimize the average AoI of the system, i.e., $\bar{\Delta}$. Let $\mathbf{Q}=$ $\left[V_{(1)}, V_{(2)}, \ldots, V_{(M)}\right]$ denote a path composed of the ground SNs, $\mathbf{t}^{\mathrm{eh}}=\left[t_{(1)}^{\mathrm{eh}}, t_{(2)}^{\mathrm{eh}}, \cdots, t_{(M)}^{\mathrm{eh}}\right]$ be the energy harvesting time vector associated with the path, and $\mathbf{t}^{\mathrm{cd}}=\left[t_{(1)}^{\mathrm{cd}}, t_{(2)}^{\mathrm{cd}}, \cdots, t_{(M)}^{\mathrm{cd}}\right]$ be the data collection time vector associated with the path. The optimization problem is mathematically expressed by

$$
\begin{aligned}
& \mathbf{P}_{\mathbf{1}}: \min _{\left(\mathbf{t}^{\mathrm{eh}}, \mathbf{t}^{\mathrm{cd}}, \mathbf{Q}\right)} \sum_{i=1}^{M} \frac{i}{M}\left(t_{(i)}^{\mathrm{eh}}+t_{(i)}^{\mathrm{cd}}+t_{(i)}^{\mathrm{f}}\right)-\frac{1}{M} \sum_{i=1}^{M} t_{(i)}^{\mathrm{eh}} \\
& \text { s.t. } \quad t_{(i)}^{\mathrm{eh}} \geq 0, t_{(i)}^{\mathrm{cd}} \geq 0, \\
& t_{(i)}^{\mathrm{cd}} \log _{2}\left(1+\frac{\gamma_{(i)} t_{(i)}^{\mathrm{eh}}}{t_{(i)}^{\mathrm{cd}}}\right) \geq \bar{D}_{(i)}, \\
& t_{(i)}^{\mathrm{f}}=d_{(i),(i+1)} v^{-1}, i=1,2, \cdots, M,
\end{aligned}
$$

where $\gamma_{(i)}=\frac{\left|h_{(i)}\right|^{2} \Phi\left(P_{r}\right)}{\sigma_{w}^{2}}$ and $\bar{D}_{(i)}=\frac{D_{(i)}}{W}$ for $i=$ $1,2, \cdots, M$. Constraint (12b) means that the total uploading data size within $t_{(i)}^{\text {cd }}$ cannot be less than the data size generated by $V_{(i)}$, and (12c) implies that the UAV's flying time is determined by the distance between the SNs.

It is difficult to directly solve Problem $\mathbf{P}_{\mathbf{1}}$, because the visiting order of all SNs is intertwined with the data collection time and the energy transfer time at each SN. To efficiently solve it, we propose a framework based on dynamic programming (DP) and ant colony (AC) approach in the next section.

\section{The Proposed Solution}

\section{A. Solution Framework}

In term of (11), the average AoI is expressed as a weighted sum of the energy transfer time and data collection time as well as the UVA's flying time. Since the energy transfer time and data collection time of at each SN are independent of the UAV's flying trajectory, Problem $\mathbf{P}_{\mathbf{1}}$ is decomposed into two sub-problems, the time allocation problem and the optimal average AoI trajectory design problem, i.e., Problem $\mathbf{P}_{\mathbf{2}}$ and Problem $\mathbf{P}_{3}$.

For Problem $\mathbf{P}_{\mathbf{2}}$, the goal is to find the optimal energy transfer time $t_{i}^{\mathrm{eh} *}$ and data collection time $t_{i}^{\mathrm{cd}}{ }^{*}$ subject to the energy and data causality constrains for $S_{i}, \forall S_{i} \in \mathbf{S}$.

$$
\begin{aligned}
& \mathbf{P}_{\mathbf{2}}: \min _{\left(t_{i}^{\mathrm{eh}}, t_{i}^{\mathrm{cd}}\right)} \sum_{i=1}^{M} t_{i}^{\mathrm{cd}}+t_{i}^{\mathrm{eh}} \\
& \text { s.t. } \quad t_{i}^{\mathrm{eh}}>0, t_{i}^{\mathrm{cd}}>0, i=1,2, \cdots, M, \\
& t_{i}^{\text {cd }} \log _{2}\left(1+\frac{\gamma_{i} t_{i}^{\text {eh }}}{t_{i}^{\text {cd }}}\right) \geq \bar{D}_{i}, i=1,2, \cdots, M,
\end{aligned}
$$

where $\gamma_{(i)}=\frac{\left|h_{(i)}\right|^{2} \Phi\left(P_{r}\right)}{\sigma_{w}^{2}}$ and $\bar{D}_{i}=\frac{D_{i}}{W}$ for $i=1,2, \cdots, M$.

It is noticed that Problem $\mathbf{P}_{\mathbf{2}}$ is actually a set of minimization problems. Each is to minimize the sum of energy transfer time and data collection time for one SN. Since the constraints are independent for different SNs, minimizing the total time of all SNs is equivalent to solving the $M$ minimization problems independently.

For Problem $\mathbf{P}_{\mathbf{3}}$, the goal is to find the optimal trajectory, visiting all the SNs in a sequence to minimize the average AoI with the obtained optimal solution $\left\{t_{i}^{\mathrm{eh} *}, t_{i}^{\mathrm{cd} *}\right\}$ to Problem $\mathbf{P}_{\mathbf{2}}$.

$$
\begin{gathered}
\mathbf{P}_{\mathbf{3}}: \min _{\mathbf{Q}} \sum_{i=1}^{M}\left[\frac{i}{M}\left(t_{(i)}^{\mathrm{eh}} * t_{(i)}^{\mathrm{cd}} * t_{(i)}^{\mathrm{f}}\right)-\frac{1}{M} t_{(i)}^{\mathrm{eh}} *\right] \\
\text { s.t. } t_{(i)}^{\mathrm{f}}=d_{(i),(i+1)} v^{-1}, i=1,2, \cdots, M .
\end{gathered}
$$

Proposition 1. The decomposition of Problem $\boldsymbol{P}_{\mathbf{1}}$ into Problem $\boldsymbol{P}_{\mathbf{2}}$ and Problem $\boldsymbol{P}_{\mathbf{3}}$ does not lose the optimality of solution to Problem $\boldsymbol{P}_{\mathbf{1}}$.

Proof. The minimal data collection time and the minimal energy transfer time required by each $\mathrm{SN}$ is independent of the flight path and its order on a path, as its required the minimal data collection time and energy transfer time are only determined by the data amount of the SN. That is to say, no matter what path the $\mathrm{SN}$ is on, its required time for data collection and energy harvesting is not changed. Therefore, one can first obtain the optimal $t_{i}^{\text {eh* }}$ and $t_{i}^{\text {cd* }}$ by solving Problem $\mathbf{P}_{2}$, and then optimize the flight path based on the obtained optimal $t_{i}^{\text {eh* }}$ and $t_{i}^{\text {cd }}{ }^{*}$. Thus, the original Problem $\mathbf{P}_{\mathbf{1}}$ can be decomposed into Problem $\mathbf{P}_{\mathbf{2}}$ and Problem $\mathbf{P}_{\mathbf{3}}$ without loss of optimality.

Based on Proposition 1, we propose a two-stage solution framework to solve Problem $\mathbf{P}_{1}$, as shown in Algorithm 1. In the first stage, the optimal $\left\{t_{i}^{\mathrm{eh}^{*}}, t_{i}^{\mathrm{cd}^{*}}\right\}$ for $i \in\{1,2, \cdots, M\}$, are found by solving Problem $\mathbf{P}_{\mathbf{2}}$. In the second stage, based on the obtained optimal $\left\{t_{i}^{\mathrm{eh}^{*}}, t_{i}^{\text {cd }}{ }^{*}\right\}$, the optimal $\mathbf{Q}^{*}$ is searched by solving Problem $\mathbf{P}_{\mathbf{3}}$. The details of solving Problem $\mathbf{P}_{\mathbf{2}}$ and Problem $\mathbf{P}_{\mathbf{3}}$ are described in the following sections.

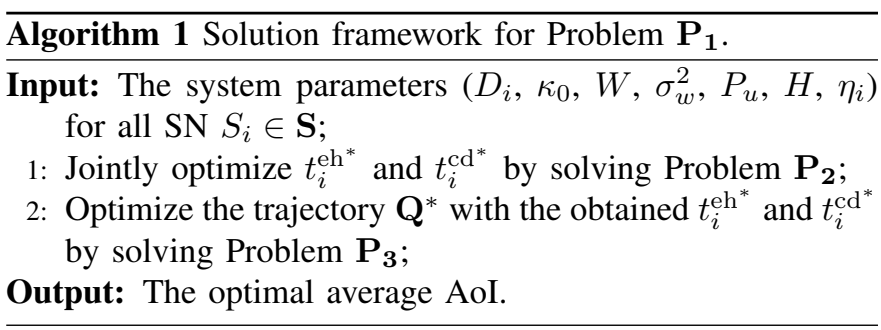

\section{B. Joint optimization of $t_{i}^{\mathrm{eh}}$ and $t_{i}^{\mathrm{cd}}$}

\section{Lemma 2. Problem $\boldsymbol{P}_{2}$ is a convex optimization problem.}

Proof. First, the objective function of Problem $\mathbf{P}_{\mathbf{2}}$ is an affine function w.r.t $\left\{t_{i}^{\text {eh }}, t_{i}^{\text {cd }}\right\}$. Second, the left hand side of constraint (13c) is a perspective function of the concave function $\log _{2}\left(1+\gamma_{i} t_{i}^{\text {eh }}\right), i \in M$. As the perspective function of concave function is also concave [38], $-t_{i}^{\mathrm{cd}} \log _{2}\left(1+\frac{\gamma_{i} \mathrm{eth}_{i}^{\mathrm{eh}}}{t_{i}^{\mathrm{cd}}}\right)$ is convex for $i=1,2, \cdots, M$. Consequently, constraint (13c) is a convex set. So, we arrive at Lemma 2.

Based on Lemma 2, Problem $\mathbf{P}_{2}$ can be solved by using convex optimization methods, and we obtain the following Lemma 3. 
Lemma 3. The optimal data collection time $t_{i}^{\mathrm{cd}}{ }^{*}$ and the optimal energy transfer time $t_{i}^{\mathrm{e}^{*}}$ to Problem $\boldsymbol{P}_{2}$ satisfy constraint (13c) with equality, i.e.,

$$
t_{i}^{\mathrm{cd} *} \log _{2}\left(1+\frac{\gamma_{i} t_{i}^{\mathrm{eh}^{*}}}{t_{i}^{\mathrm{cd}}}\right)=\bar{D}_{i}, i=1,2, \cdots, M .
$$

Proof. By observing (13c), one can find that the left hand side of (13c) is a monotonically increasing function in both $t_{i}^{\text {cd }}$ and $t_{i}^{\mathrm{eh}}$. When the constraint (13c) takes " $>$ ", $t_{i}^{\mathrm{eh}}$ and $t_{i}^{\mathrm{cd}}$ can be reduced to further decrease the system AoI. Therefore, the optimal energy transfer time and data collection time satisfy (15).

Based on Lemma 3, we have the following theorem.

Theorem 1. The optimal time assigned to collect data and charge energy for $S_{i}$, i.e., $\left\{t_{i}^{\mathrm{eh}^{*}}, t_{i}^{\text {cd }}{ }^{*}\right\}$ satisfy

$$
t_{i}^{\mathrm{cd}^{*}}=\frac{\ln 2 \bar{D}_{i}}{\mathcal{W}\left(\frac{\gamma_{i}-1}{e}\right)+1}, i=1,2, \cdots, M,
$$

and

$$
t_{i}^{\mathrm{eh}^{*}}=\frac{\left(2^{\left.\frac{\overline{\bar{D}_{i}}}{\mathrm{t}_{i}^{\mathrm{d} *}}-1\right) t_{i}^{\mathrm{cd}}}\right.}{\gamma_{i}}, i=1,2, \cdots, M,
$$

respectively, where $\mathcal{W}(\cdot)$ is the Lambert W function [39].

Proof. The Lagrangian of Problem $\mathbf{P}_{\mathbf{2}}$ is

$$
\begin{aligned}
L\left(t_{i}^{\mathrm{eh}}, t_{i}^{\mathrm{cd}}, \mu_{i}\right)= & \sum_{i=1}^{M} t_{i}^{\mathrm{cd}}+t_{i}^{\mathrm{eh}} \\
& +\sum_{i=1}^{M} \mu_{i}\left[\bar{D}_{i}-t_{i}^{\mathrm{cd}} \log _{2}\left(1+\frac{\gamma_{i} t_{i}^{\mathrm{eh}}}{t_{i}^{\mathrm{cd}}}\right)\right]
\end{aligned}
$$

where, $\mu_{i}$ is the non-negative Lagrangian dual variable associated with the constraint (13c). Applying KKT conditions and (15), we have that

$$
\begin{aligned}
& \frac{\partial L}{\partial t_{i}^{\mathrm{cd}}}=1-\mu_{i}^{*}\left[\frac{\bar{D}_{i}}{t_{i}^{\mathrm{cd}}}-\frac{1}{\ln 2} \cdot \frac{2^{\frac{\bar{D}_{i}}{t_{i}^{\mathrm{d}^{*}}}}-1}{2^{\frac{\bar{D}_{i}}{t_{i}^{\mathrm{cd}}}}}\right]=0, \\
& \frac{\partial L}{\partial t_{i}^{\text {eh }}}=1-\mu_{i}^{*}\left[\frac{1}{\ln 2} \cdot \frac{\gamma_{i}}{2^{\frac{D_{i}}{t_{i}^{c *}}}}\right]=0,
\end{aligned}
$$

with $i \in\{1,2,3, \cdots, M\}$. By solving (19) and (20), thus Theorem 1 can be proved.

\section{Optimization of $\mathbf{Q}$ with the obtained $t_{i}^{\mathrm{e}^{*}}$ and $t_{i}^{\mathrm{cd}}{ }^{*}$}

Lemma 4. Problem $\boldsymbol{P}_{\mathbf{3}}$ is an NP-hard problem.

Proof. Given $M$ SNs and the optimal data collection time and the optimal energy transfer time of each $\mathrm{SN}$ and the distance each pair of SNs, i.e., $\left[t_{1}^{\mathrm{cd}^{*}}, t_{2}^{\mathrm{cd}}, \ldots, t_{M}^{\mathrm{cd}}\right]$, $\left[t_{1}^{\mathrm{eh}^{*}}, t_{2}^{\mathrm{eh}^{*}}, \ldots, t_{M}^{\mathrm{eh}^{*}}\right]$ and $d_{i, j}, \forall i \in\{0,1,2, \ldots, M\}$. Problem $\boldsymbol{P}_{3}$ aims to find the shortest path that starts from $S_{0}$ and visits each SN once and returns $S_{0}$. According to [42], if we can reduce a well-known NP-Hard problem to Problem $\boldsymbol{P}_{3}$, Problem $\boldsymbol{P}_{\mathbf{3}}$ can be proved to be NP-Hard. As is known, the traveling salesman problem (TSP) is a NP-hard problem [43], which aims to find the shortest loop to visit each city once and return to the starting city for a given set of cities and the distances between any two of them. If we map each city in the TSP problem to a SN with $\left[t_{i}^{\mathrm{eh}^{*}}, t_{i}^{\mathrm{cd}}{ }^{*}\right]$, the TSP problem is reduced to our considered Problem $\boldsymbol{P}_{\mathbf{3}}$. Thus, $\boldsymbol{P}_{\mathbf{3}}$ is an NP-Hard problem.

As the feasible set of the trajectory is composed of vectors formed by the sequence of the visiting SNs, and Problem $\mathbf{P}_{\mathbf{3}}$ is an NP-hard problem, it cannot be solved by general convex optimization theory. Therefore, we design two algorithm to solve it. The first one is the DP-based algorithm, which finds the global optimal solution by checking all candidate solutions and may be too complex, especially, where $M$ is relatively large. So the second one algorithm, i.e., the ACbased algorithm, is presented, as an alternative approach.

1) DP-based algorithm: In order to solve Problem $\mathbf{P}_{\mathbf{3}}$ based on DP, we define the time interval from the moment that UAV starts to collect data from $V_{(i)}$ to the moment that the UAV starts to collect data from $V_{(i+1)}$ as the average AoI increment, i.e.,

$$
\Delta_{L_{(i),(i+1)}}=\frac{i}{M}\left(t_{(i)}^{\mathrm{cd} *}+t_{(i)}^{\mathrm{eh} *}+t_{(i)}^{\mathrm{f}}\right)-\frac{1}{M} t_{(i)}^{\mathrm{eh}},
$$

which is the time interval between $U_{i}$ to $U_{i+1}$.

Let $C\left(V_{(i)}\right)$ represent the minimum AoI cost of the path starting from $V_{(i)}$, passing all the rest SNs exactly once and returning back to the data center $S_{0}$. Then, the minimum average AoI cost is given by

$$
C\left(V_{(i)}\right)=\left\{\begin{array}{cc}
\Delta_{L_{(M),(M+1)}}, & i=M ; \\
\min \left\{\Delta_{L_{(i),(i+1)}}+C\left(V_{(i+1)}\right)\right\}, & \\
i=1,2, \cdots, & M-1 .
\end{array}\right.
$$

Let $\widehat{\mathbf{S}}$ represent the set composed of the SNs that are still not visited. Based on (21) and (22), a DP-based algorithm is presented, as shown in Algorithm 2.

Algorithm 2 DP-based trajectory planning algorithm.

Input: The location of all SNs, the optimal data collection time $t_{i}^{\text {cd }}{ }^{*}$ and energy transfer time $t_{i}^{\text {eh* }}$ of $S_{i}$, for $i=$ $1,2, \cdots, M$.

1: Calculate the average AoI increment $\Delta_{L_{(i),(i+1)}}$ of data collected from $V_{(i)}$ to collect data to $V_{(i+1)}$ in terms of (21);

2: for $m=1$ to $M$ do

3: $\quad$ Initialize $i=1, V_{(i)}=S_{m}$, and $\widehat{\mathbf{S}}=\mathbf{S}$;

4: $\quad$ while $i \leq M$ do

5: $\quad$ Calculate $\widehat{\mathbf{S}}=\widehat{\mathbf{S}}-\left\{V_{(i)}\right\}$;

6: $\quad$ Calculate the minimum average AoI cost $C\left(V_{(i)}\right)$ according to $(22)$;

7: $\quad$ Update $i$ by $i=i+1$;

8: $\quad$ end while

9: end for

10: Add $V_{(0)}=V_{(M+1)}=S_{0}$ to the paths and find the minimum average AoI $\bar{\Delta}$;

11: Trace back to find the optimal path starting with the data center $S_{0}$ and ending with the data center $S_{0}$;

Output: The optimal flying trajectory $\mathbf{Q}^{*}$. 
It can be seen that DP-based algorithm actually finds the optimal trajectory by calculating the AoI cost $C\left(V_{(i)}\right)$ and comparing the age costs for all SNs. The DP-based algorithm finds the optimal solution for each sub-problem in an iterative manner, so the optimal solution to the original problem can be derived. However, the computational complexity of the DP-based algorithm is about $O\left(2^{M} M^{2}\right)$. That is, the time efficiency of the algorithm may be poor, and the computational complexity will increase dramatically as the problem size becomes larger.

2) AC-based algorithm: As is known, ant colony (AC) algorithm adopts positive feedback mechanism, which achieves good parallelism, cooperation, and robustness. Moreover, it also has good searchability and strong dynamics, so it is often used in track planning. The detailed information about AC algorithm can be found in [44]. Here, we also present an algorithm to solve Problem $\mathbf{P}_{\mathbf{3}}$ based on AC.

The basic idea is that according to the habit, ants leave pheromones along the path during the foraging process. The shorter the path, the more pheromones remain, and the ants are more likely to choose the path. This leads to more and more pheromones on the optimal path, thus forming a positive feedback mechanism to promote the system to find the optimal path. However, in the process of constructing the solution of AC algorithm, the random selection strategy slows down the evolution of the algorithm. To reduce the probability of the $\mathrm{AC}$ algorithm to a certain extent stuck in local optimum solution and improve the convergence performance of the AC algorithm, we introduce the pseudo-random proportional rule into the standard AC algorithm [45]. For clarity, the presented AC-based algorithm is summarized in Algorithm 3, where the detailed process of its five steps are explained as follows.

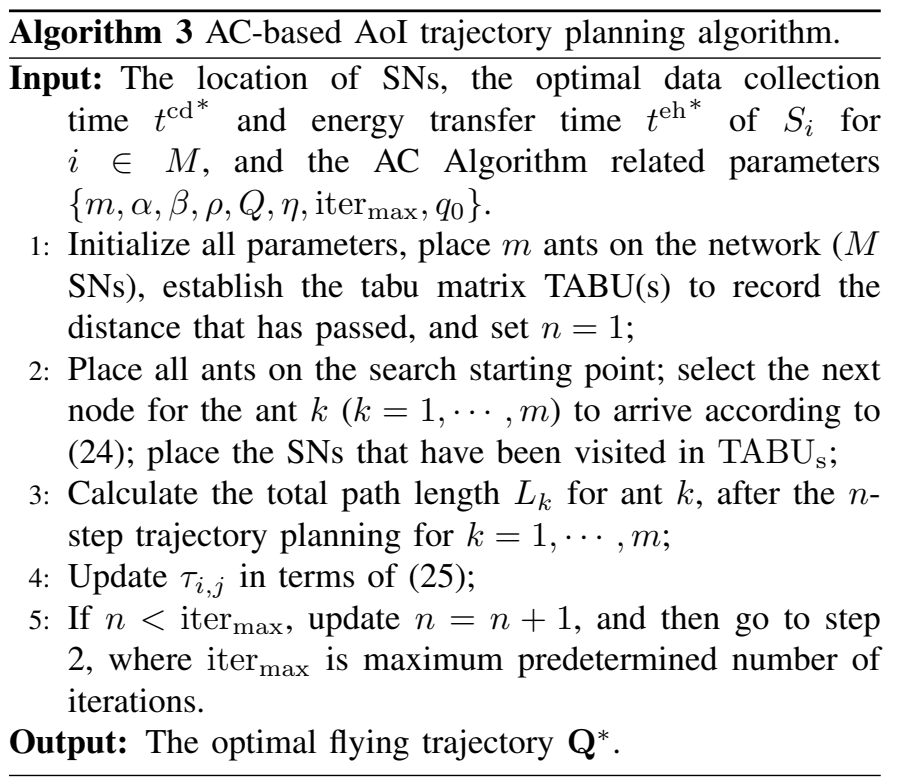

Step 1: Initialize all parameters, place $m$ ants on the network ( $M \mathrm{SNs}$ ), establish the tabu matrix $\mathrm{TABU}_{\mathrm{s}}$ to record the distance that has passed, and set $n=1$.

Step 2: Place all ants on the search starting point, and the ant $k(k=1, \cdots, m)$ selects the next node to arrive according to the pseudo-random proportional rule. Let $P_{i, j}^{k}(t)$ indicate the probability that ant $k$ transfers from $S_{i}$ to $S_{j}$ at time $t$,

$$
P_{i j}^{k}(t)=\left\{\begin{array}{cl}
\frac{\left[\tau_{i, j}(t)\right]^{\alpha}\left[\eta_{i, j}(t)\right]^{\beta}}{\sum_{j \notin \operatorname{TABU}_{\mathrm{s}}\left[\tau_{i, j}(t)\right]^{\alpha}\left[\eta_{i, j}(t)\right]^{\beta}},}, j \in \text { allowed }_{\mathrm{k}} & \text { other, }
\end{array}\right.
$$

where $\tau_{i, j}(t)$ represents the amount of pheromone content on the path connect $S_{i}$ and $S_{j}$ at time $t . \eta_{i, j}(t)$ is a heuristic function, which represents the visibility of the ant to the path, with $\eta_{i, j}(t)=\frac{1}{\Delta L_{i, j}}$ and $\Delta L_{i, j}=t_{i}^{\mathrm{cd}^{*}}+d_{i, j} v^{-1}+t_{j}^{\mathrm{eh}}$. $\alpha$ is the pheromone importance factor and $\beta$ is the expected heuristic factor. The set allowed $\mathrm{k}_{\mathrm{k}}=\left\{\mathrm{S}-\mathrm{TABU}_{\mathrm{s}}\right\}$ represents the available SNs that can be selected next time by ant $k$. The SNs that have been visited are placed in $\mathrm{TABU}_{\mathrm{s}}$, and the initial moment are set $t=0$.

The pseudo-random proportional rule is given by [45]:

$$
j= \begin{cases}\arg \max _{\mathrm{j} \in \text { allowed }}\left[\tau_{\mathrm{i}, \mathrm{j}}(\mathrm{t})\right]^{\alpha}\left[\eta_{\mathrm{i}, \mathrm{j}}(\mathrm{t})\right]^{\beta}, & q \leq q_{0} \\ j \text { from }(23) & , \text { other }\end{cases}
$$

where $q$ is a random variable uniformly distributed in the interval $[0,1]$, and $q_{0} \in[0,1]$ is a parameter that represents the probability that the ant chooses the current best possible movement mode.

Step 3: After each ant has completed the $n$-step trajectory planning, i.e., all ants arriving at the target point from the start point, calculates the total path length $L_{k}$ for $k=1,2, \cdots, m$.

Step 4: Each ant updates the pheromone, i.e., $\tau_{i, j}$, according to

$$
\begin{gathered}
\tau_{i, j}(t+n)=(1-\rho) \tau_{i, j}(t)+\Delta \tau_{i, j}(t), \\
\Delta \tau_{i, j}(t)=\sum_{k=1}^{m} \Delta \tau_{i, j}^{k}(t),
\end{gathered}
$$

where $\rho$ represents pheromone volatilization coefficients, and $(1-\rho)$ represents pheromone residual factor, and $\Delta \tau_{i, j}^{k}(t)$ is the increment of pheromone on path $\left(S_{i}, S_{j}\right)$ in current iteration. Particularly, at the initial time, $\Delta \tau_{i, j}^{k}(0)=0$, and

$$
\Delta \tau_{i, j}^{k}(t)=\left\{\begin{array}{cl}
\frac{Q}{L_{k}}, & \text { if ant } k \text { passed though } \operatorname{SNs}\left(S_{i}, S_{j}\right) \\
0, & \text { otherwise. }
\end{array}\right.
$$

where $Q$ the pheromone concentration.

Step 5: If $n \geq$ iter $_{\max }$, algorithm stops. Otherwise, if $n<$ iter $_{\max }$, update $n=n+1$, and then go to step 2, where iter $_{\max }$ is the predetermined maximum number of iterations.

\section{NUMERICAL RESULTS}

This section provides some simulation results to discuss the performance of our proposed algorithms. We simulated a UAVassisted wireless powered IoT network that consists of one data center, one UAV and $M$ SNs. The SNs are randomly distributed in a circular area with a radius of $3000 \mathrm{~m}$. The data center is located at the origin $(0,0)$. The flight height $H$ and speed $v$ of the UAV are set as $10 \mathrm{~m}$ and $20 \mathrm{~m} / \mathrm{s}$, respectively. The UAV's transmit power is set as $P_{u}=0.5 \mathrm{~W}$ and the data size is set as $D_{i}=1 \mathrm{Mbits}$. The system bandwidth is set as $W=1 \mathrm{MHz}$ and the path loss factor is set as $\kappa=2$. The channel power gain at the reference distance $1 \mathrm{~m}$ is set 


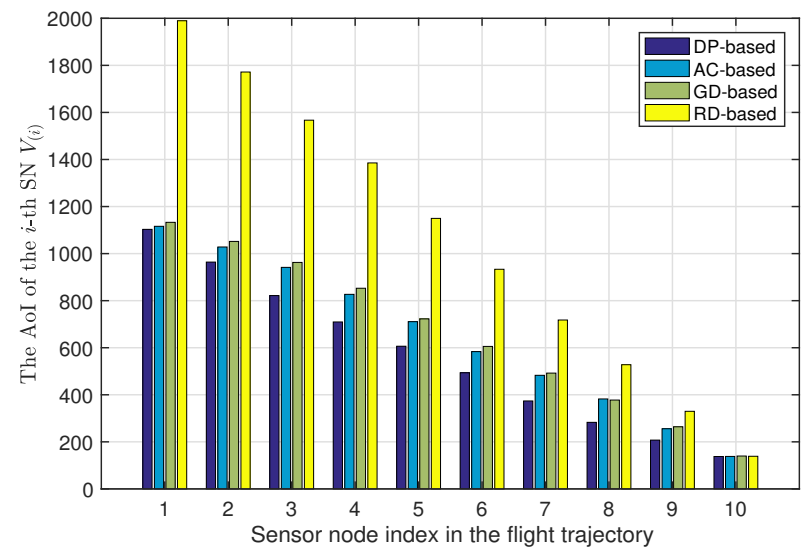

Fig. 3. The AoI $\Delta_{(i)}$ of $V_{(i)}$ in the different trajectories.

as $\kappa_{0}=-50 \mathrm{~dB}$ and the noise power $\sigma_{w}^{2}=-110 \mathrm{dBm}$ [46]. For the non-linear EH model, we set $P_{\max }$ as $24 \mathrm{~mW}$ which corresponds to the maximum harvested power at each $\mathrm{SN}$. Besides, we adopt $\mathrm{a}=4$ and $\mathrm{b}=0.001$. The $\mathrm{AC}$ related parameters are set as $m=M-2, \alpha=1, \beta=5, \rho=0.5$, $Q=100$, iter $\max =30$, and $q_{0}=0.9$. For comparison, the greedy (GD)-based algorithm and the random (RD)-based algorithm are simulated as the benchmark methods. In the GDbased algorithm, it starts from $S_{0}$ to find the nearest $\mathrm{SN}$, and the process repeats until all $M$ SNs are found. In the RDbased algorithm, a random flight trajectory that visits all SNs is adopted, i.e., $\mathbf{Q}=\left[S_{1}, S_{2}, \cdots, S_{M}\right]$. Suppose that the UAV takes off at time $t=0$.

Fig. 3 plots the AoI $\Delta_{(i)}\left(t=U_{t}\right)$ of each $V_{(i)}$ achieved by the four algorithms with $M=10$. The simulation result shows that compared with the RD-based method, our proposed algorithms and the GD-based algorithm achieve much smaller AoI for each SN. It implies that by optimally panning the trajectory and allocating time, the AoI associated with each SN can be greatly decreased. The reason is that if the AoI of $V_{(i)}$ is relatively large, it will enlarge the AoI of the data collected before accessing $V_{(i)}$. Therefore, by optimizing trajectory, the AoI of $S_{i}$ is reduced greatly. In order to clearly observe the benefits brought by optimizing the UAV trajectory, Fig. 4 plots the ratio of the AoI $\Delta_{(i)}\left(t=U_{t}\right)$ of each $V_{(i)}$ achieved by the three algorithms normalized to the AoI achieved by RD-based. It shows that the proposed algorithms and GD-based algorithm reduce the AoI of data collected from the first eight SNs by $50 \%-72 \%$ of that achieved by the RD-based algorithm.

Fig. 5 plots four UAV trajectories achieved by the DPbased, the AC-based, the GD-based and RD-based algorithms. Clearly they look different. However, we see that the AC-based algorithm finds a trajectory very similar to that by the DPbased algorithm, which shows that the AC-based heuristic can be very close to the optimal.

In order to clearly compare the average AoI achieved by the DP-based, the AC-based and the GD-based algorithms, Fig. 6 is presented. It is seen that among the three algorithms, the DPbased algorithm achieves the smallest AoI, because it is able to find the optimal average AoI trajectory by comparing all

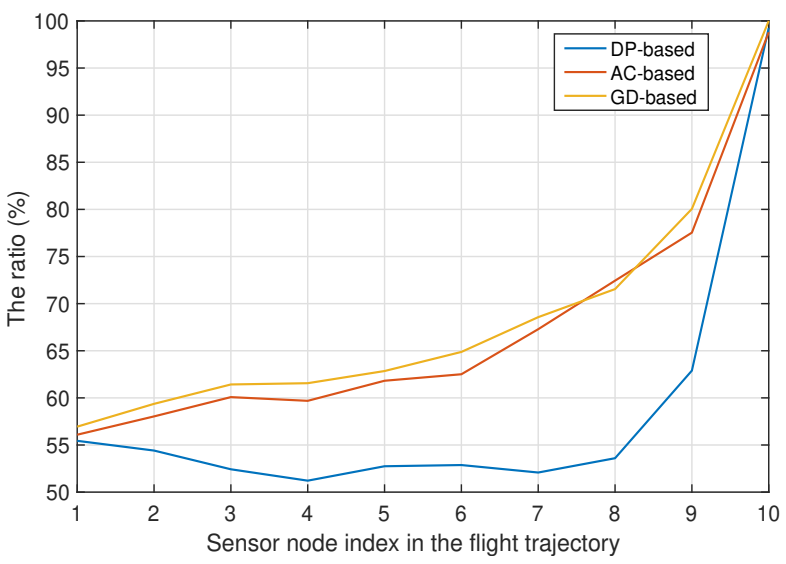

Fig. 4. The ratio of the AoI $\Delta_{(i)}$ of each SN $V_{(i)}$ achieved by the three algorithms to the AoI achieved by RD-based.

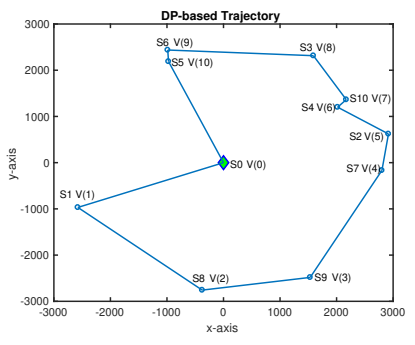

(a) DP-based algorithm

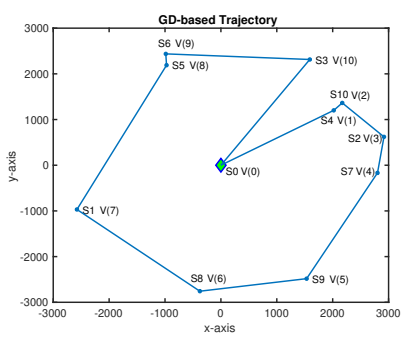

(c) GD-based algorithm

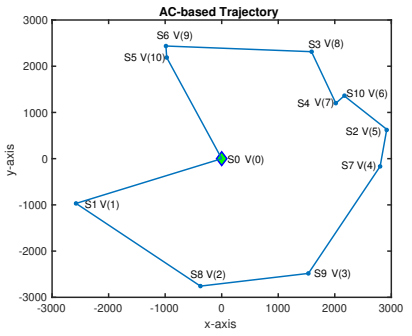

(b) AC-based algorithm

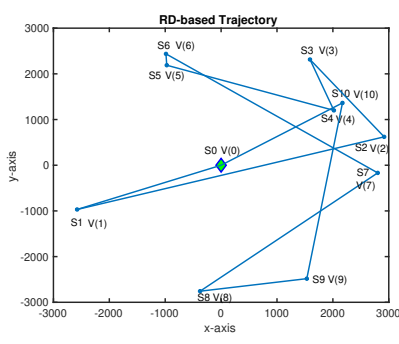

(d) RD-based algorithm
Fig. 5. An example of four trajectories: (a) DP-based algorithm; (b) AC-based algorithm; (c) GD-based algorithm; (d) RD-based algorithm.

the candidate paths. The AC-based algorithm achieves the near optimal solution to the DP-based one. The GD-based algorithm achieves the highest AoI, as it finds the local optimal solution each time, which is not necessarily the global optimal solution.

Fig. 7 shows the AoI $\Delta_{(i)}\left(t=U_{t}\right)$ of each $V_{(i)}$ achieved by the three algorithms under different data size, i.e., $D_{i}=$ 1 Mbits and $D_{i}=3$ Mbits, $\forall i \in\{1,2, \cdots, M\}$. One can observe that with the increase of data size $D_{i}$, the AoI of $V_{i}$ increases, but the differences in the AoI obtained by the three algorithms remain almost the same regardless of the data size. The reason may be that the larger $D_{i}$ is, the longer the data collection time $t_{i}^{\text {cd }}$ and the longer the energy transfer time $t_{i}^{\text {eh }}$, but this may not charge the visiting order obtained by three algorithms.

Fig. 8 shows the average AoI achieved by the three al- 


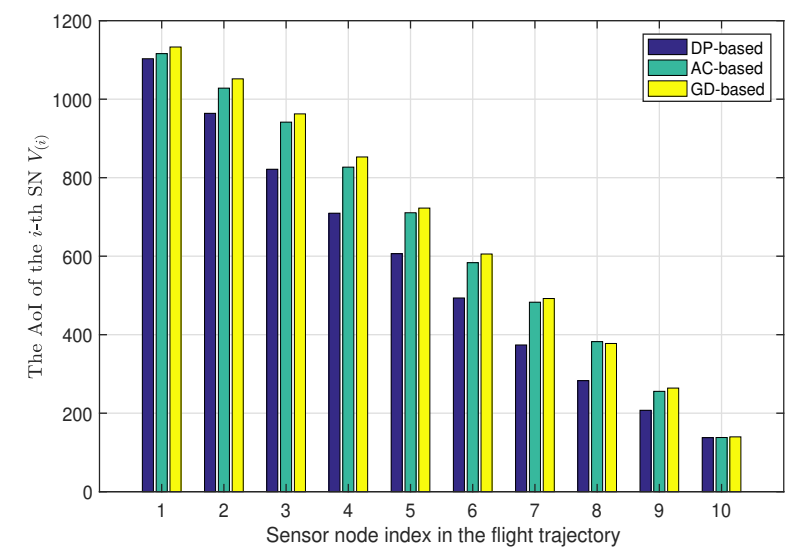

Fig. 6. The AoI $\Delta_{(i)}$ of SN $V_{(i)}$ in the different trajectories.

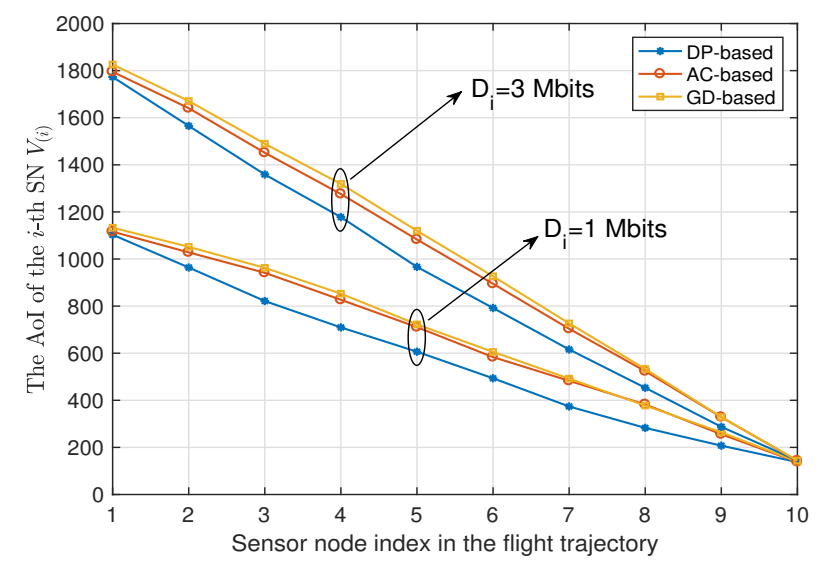

Fig. 7. The AoI $\Delta_{(i)}$ of $\mathrm{SN} V_{(i)}$ in the different trajectories.

gorithms versus the transmit power of the UAV. One can observe that with the increase of transmit power at UAV, the average AoI gradually decreases and eventually tends to be flat, because more transmit energy, lower energy harvesting time and data collection time, but according to Shannon theorem, the information transmission rate cannot be increased infinitely by increasing the transmit power. So, the AoI cannot be decreased to zero by increasing the transmit power of UAV. In addition, the average AoI achieved by three algorithms with $M=10$ are larger than that with $M=6$. Because the more SNs, the longer flying trajectory and the more data required to be collected, resulting in large average AoI.

In order to provide deeper insights about the effect of $M$ on the average AoI, the average AoI versus the number of SNs achieved by the AC-based and the GD-based algorithms are plotted in Fig. 9, where more SNs are deployed in the system. It is shown that the average AoI roughly linear increases with the number of SNs, and it also shows that the AoI achieved by the AC-based algorithm decreased by about $5 \%$ of the AoI achieved by GD-based algorithm.

In addition, the running time and peak memory of the AC-based and the GD-based algorithms versus the number of SNs are shown in Table I and Table II, respectively.

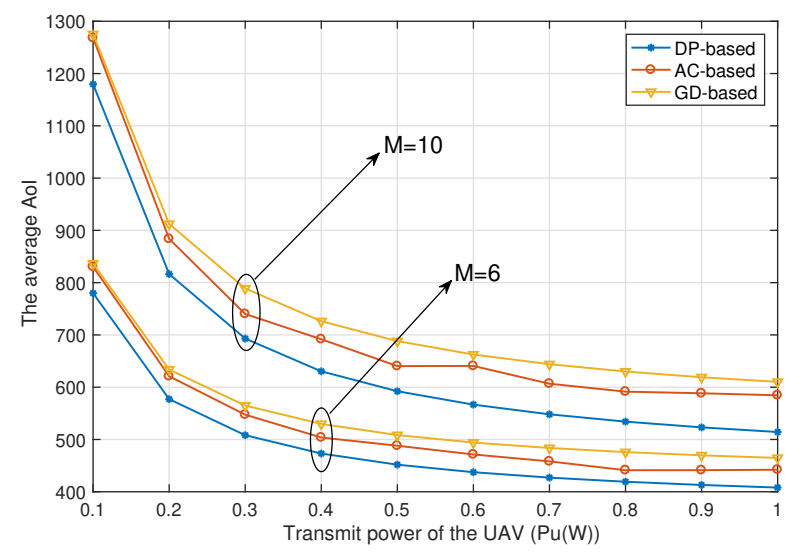

Fig. 8. The average AoI $\bar{\Delta}$ versus the transmit power of the UAV.

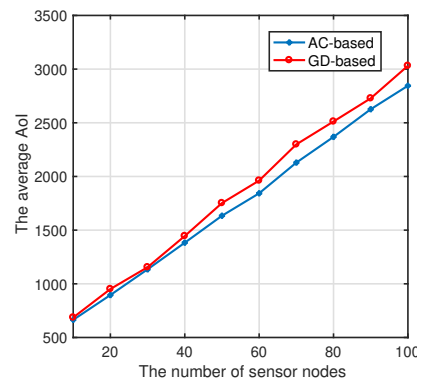

(a) The average AoI versus $M$.

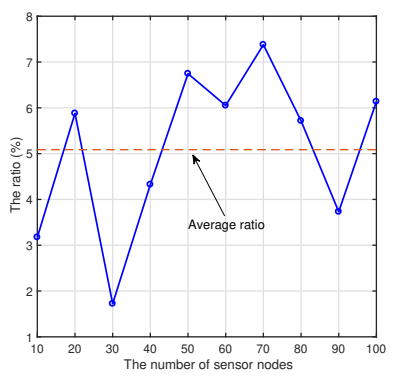

(b) The ratio versus $M$.
Fig. 9. The average AoI and the ratio of AoI versus the number of SN $M$.

It can be seen that as the number of SNs increases, the running time and peak memory of the AC-based and the GD-based algorithms increase. Moreover, compared with GDbased algorithm, AC-based algorithm consumes more time and more memory. Combined with Fig. 9, it can be inferred that if the computing resources of the system is relatively abundant, AC-based algorithm should be selected to achieve the smaller AoI. Otherwise, GD-based one may be a better choice to save computing resources by sacrificing some AoI performance.

Fig. 10 depicts the average AoI versus the UAV's altitude under the non-linear EH model and the linear EH model. In the simulations, the UAV's trajectory is obtained by DP-based algorithm. It is noticed that in the simulations, the parameters associated with the non-linear EH model are set according to [47], [48], which were obtained by measuring practical EH circuits. Moreover, the maximum conversion efficiency of a EH circuit is limited by factors such as device parasitics, harmonic generation, and impedance matching, resulting in a maximum conversion efficiency of less than $90 \%$ [49]. That is, in practice, $\eta_{i}$ is not more than 0.9. Thus, we provide some simulation results on linear EH model for comparison, where $\eta_{i}=0.3,0.6$ and 0.9 in Fig. 10. One can observe that a smaller average AoI is obtained under the non-linear EH model than under the linear EH model, because the linear EH model mismatches the non-linear feature of the system, resulting in inaccurate optimization result. It is seen when $\eta_{i}=0.9$, the nonlinear EH model still outperforms the linear one. And when 
TABLE I

THE RUNNING TIME OF THE ALGORITHMS

\begin{tabular}{lllllllllll}
\hline & $M=10$ & $M=20$ & $M=30$ & $M=40$ & $M=50$ & $M=60$ & $M=70$ & $M=80$ & $M=90$ & $M=100$ \\
\hline AC-based & $0.1013 \mathrm{~s}$ & $0.2522 \mathrm{~s}$ & $0.5012 \mathrm{~s}$ & $0.9103 \mathrm{~s}$ & $1.4873 \mathrm{~s}$ & $2.0702 \mathrm{~s}$ & $2.9640 \mathrm{~s}$ & $4.1815 \mathrm{~s}$ & $5.3782 \mathrm{~s}$ & $6.9601 \mathrm{~s}$ \\
GD-based & $0.0133 \mathrm{~s}$ & $0.0138 \mathrm{~s}$ & $0.0143 \mathrm{~s}$ & $0.0145 \mathrm{~s}$ & $0.0147 \mathrm{~s}$ & $0.0149 \mathrm{~s}$ & $0.0152 \mathrm{~s}$ & $0.0157 \mathrm{~s}$ & $0.0163 \mathrm{~s}$ & $0.0168 \mathrm{~s}$ \\
\hline
\end{tabular}

TABLE II

THE PEAK MEMORY OF THE ALGORITHMS

\begin{tabular}{lllllllllll}
\hline & $M=10$ & $M=20$ & $M=30$ & $M=40$ & $M=50$ & $M=60$ & $M=70$ & $M=80$ & $M=90$ & $M=100$ \\
\hline AC-based & $208.91 \mathrm{~Kb}$ & $275.45 \mathrm{~Kb}$ & $287.45 \mathrm{~Kb}$ & $301.14 \mathrm{~Kb}$ & $323.44 \mathrm{~Kb}$ & $353.95 \mathrm{~Kb}$ & $513.47 \mathrm{~Kb}$ & $748.18 \mathrm{~Kb}$ & $860.34 \mathrm{~Kb}$ & $968.20 \mathrm{~Kb}$ \\
GD-based & $11.72 \mathrm{~Kb}$ & $21.16 \mathrm{~Kb}$ & $34.97 \mathrm{~Kb}$ & $45.62 \mathrm{~Kb}$ & $49.75 \mathrm{~Kb}$ & $52.74 \mathrm{~Kb}$ & $54.86 \mathrm{~Kb}$ & $67.14 \mathrm{~Kb}$ & $78.92 \mathrm{~Kb}$ & $81.16 \mathrm{~Kb}$ \\
\hline
\end{tabular}

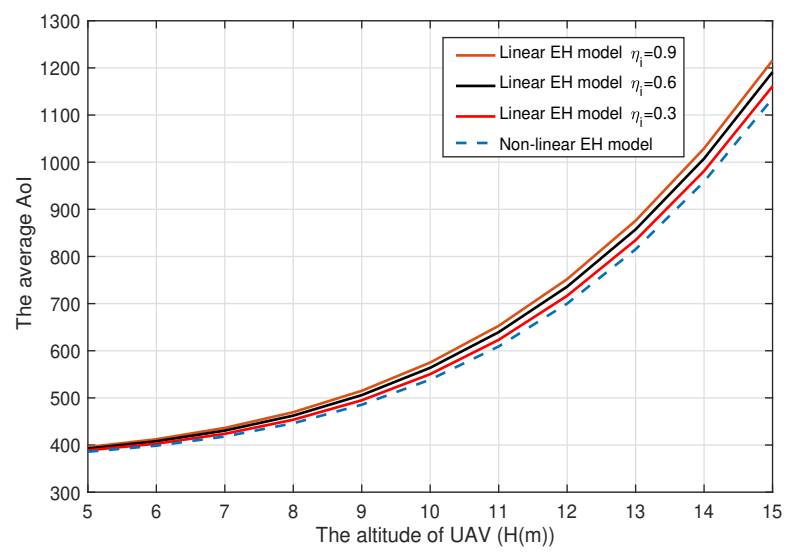

Fig. 10. The average AoI versus the flight altitude of the UAV.

$\eta_{i}=0.3$, it is closer to the result obtained by the non-linear EH model.

To clearly show the non-linear EH model and the linear EH model with different energy harvesting efficiency $\eta_{i}$, Fig. 11 is presented. In the simulations, the UAV's flight height $H$ is set as $10 \mathrm{~m}$. It is observed that, compared with the non-linear EH model, the higher the value of the conversion efficiency $\eta_{i}$, the larger the bias of the output power caused by the linear EH model. That is to say, linear EH model with higher energy harvesting efficiency $\eta_{i}$ may give a wrong AoI expectation, but exactly results in the worse AoI performance (i.e., the higher AoI). Fig. 10 also shows that the larger $H$, the larger the achieved average AoI. Moreover, the difference between the average AoI obtained with the linear EH model and the non-linear EH model also increases with the increment of $H$. The reason can be explained by Fig. 12, where it is shown that as $H$ increases, it requires longer time to harvest energy. That is, even if the output power difference between the two $\mathrm{EH}$ models decreases with the increase of $H$, the required time for energy harvesting increases, resulting in the larger gap between the two curves in Fig. 10.

Fig. 13 plots the average AoI versus the transmit power of the UAV with the equal time allocation and our proposed optimal time allocation. In the simulations, the data size of all SNs are different, i.e., $D_{i}=0.1+0.2(i-1)$ Mbits for $i=1,2, \cdots, M$. For the equal time allocation, the data collect

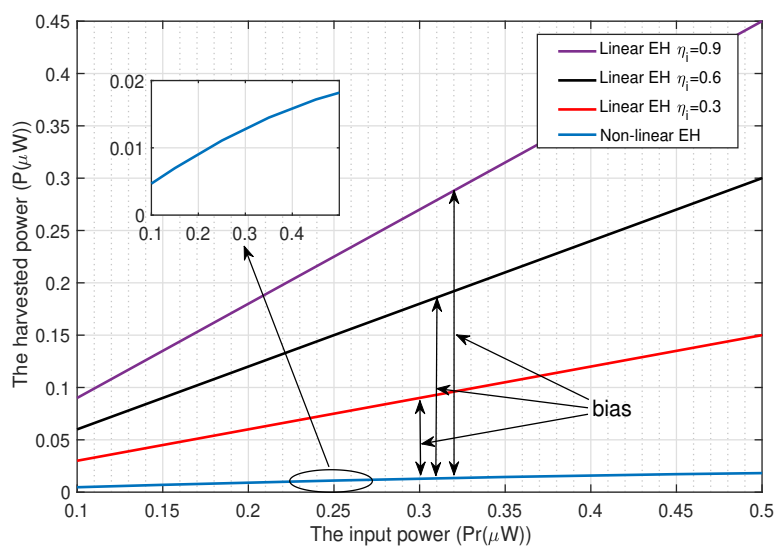

Fig. 11. The harvested power versus the input power.

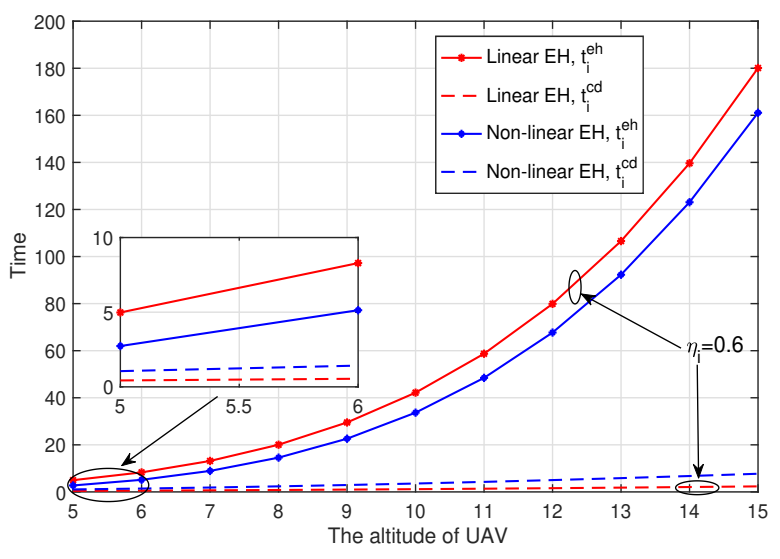

Fig. 12. Time versus the flight altitude of the UAV.

time is set as $t_{i}^{\text {cd }}=t_{2}^{\text {cd }}$ for all $i=1,2, \cdots, M$. It is observed that the average AoI decreases with the increment of the transmit power of UAV and finally tends to be stable, and the gap between the two lines also decreases with the increment the transmit power of UAV. This indicates that optimization of the energy transfer time and the data collection time is helpful in reducing the system average AoI, and the effect of the time allocation on the average AoI seems unchanged versus the transmit power of the UAV. From Fig. 14, one can observe 


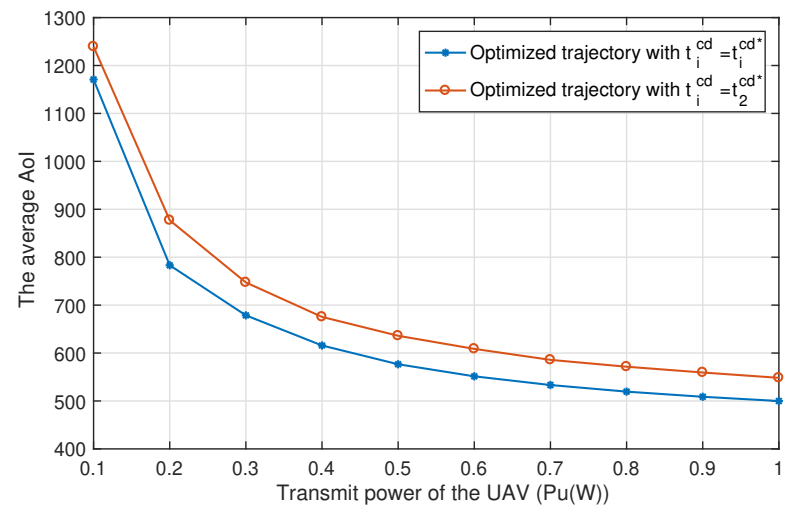

Fig. 13. The average AoI versus transmit power of the UAV.

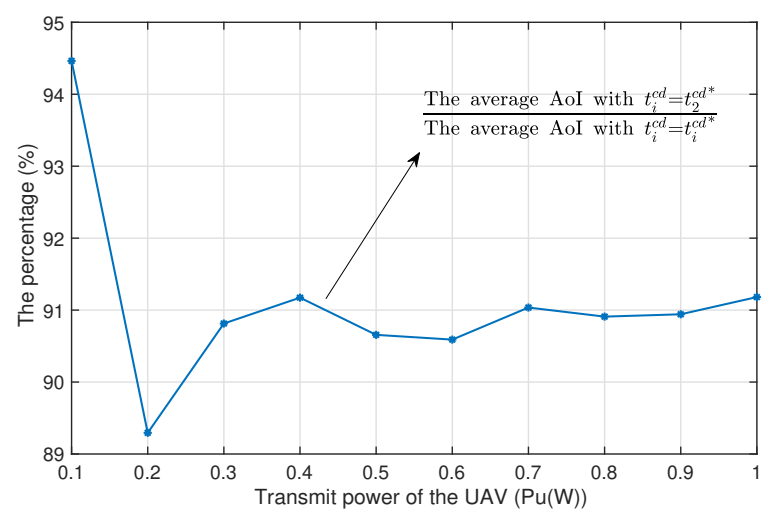

Fig. 14. The percentage of AoI obtained at the optimization time to AoI obtained at the equal time versus transmit power of the UAV.

that the average AoI obtained with optimized data collection time and energy harvest time can be reduced to about $91 \%$ of the average AoI obtained with equal time collection data.

Fig. 15 plots the average AoI versus the data size, where the optimal trajectory is obtained by the DP-based algorithm. One can observe that the average AoI roughly linearly increases with the data size. To discuss the effect of the energy harvesting time on the average AoI,

Fig. 16 plots the average energy transfer time versus the

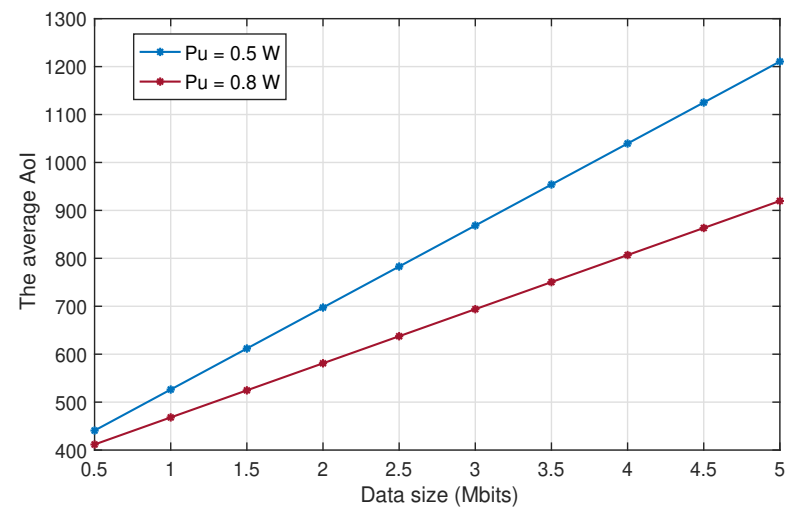

Fig. 15. The average AoI versus transmit power of the $\operatorname{UAV}\left(P_{u}\right)$.

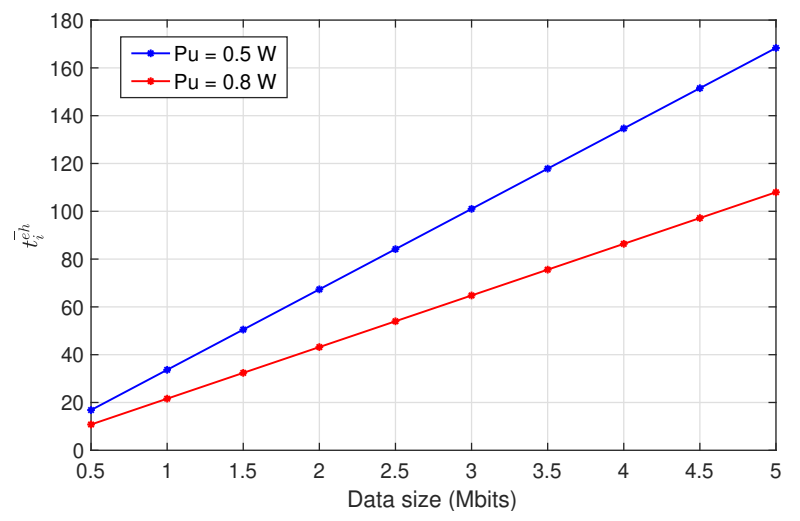

Fig. 16. The average energy harvest time $t_{i}^{\overline{\mathrm{eh}}}$ versus the data size.

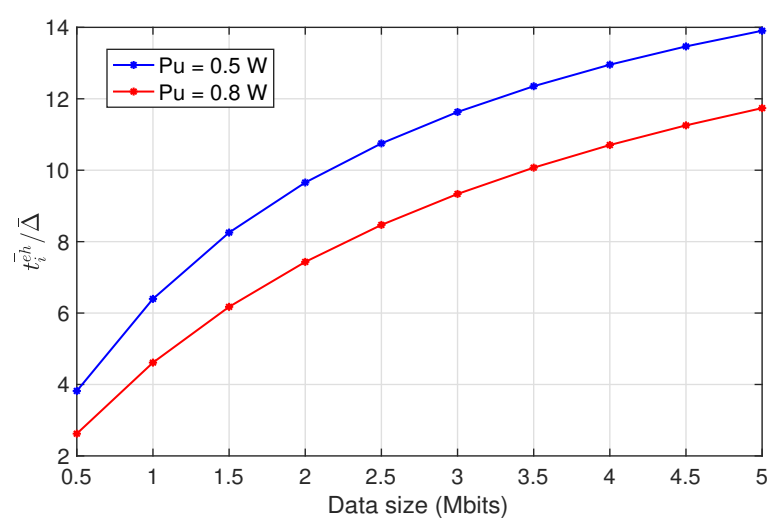

Fig. 17. The percentage of the average energy harvest time to the average AoI $t_{i}^{\overline{e h}} / \bar{\Delta}$ versus the data size.

data size $D_{i}$ with $P_{u}=0.5 \mathrm{~W}$ and $P_{u}=0.8 \mathrm{~W}$, and Fig. 17 plots the proportion of the energy transfer time in the average AoI versus the data size $D_{i}$ with $P_{u}=0.5 \mathrm{~W}$ and $P_{u}=0.8$ W. In the simulations, the UAV's trajectory is obtained by DPbased algorithm. It can be seen that the average energy transfer time increases with the increase of data size and decreases with the increases of transmit power of UAV. Moreover, the proportion of the energy transfer time in the average AoI increases logarithmically as the data size increases.

\section{CONClusion}

This paper studied the UAV-assisted wireless powered IoT system. We formulated an optimization problem to minimize the average AoI of the data collected from all ground SNs by jointly optimizing the UAV's trajectory and energy transfer and data collection time for each SN. In order to solve this problem, we proposed an efficient algorithm framework. Simulation results shows that the DP-based algorithm obtains the minimal average AoI of the system, and the AC-based and the GD-based algorithms finds the near-optimal average AoI. When the number of SNs is large, the AC-based algorithm is the best choice. Moreover, compared with traditional linear EH model, employing the non-linear EH model can decrease the average AoI as the real systems are operated based on the EH circuits with non-linear features. Besides, as the flying altitude 
of the UAV increases, the average AoI increases, and the difference between the average AoI obtained by the linear EH model and the non-linear EH model also increases. Moreover, the average AoI increases basically linearly versus the data size. These conclusions may be helpful to keep the SNs data fresh in wireless sensor networks.

\section{REFERENCES}

[1] Q. Cui, Y. Wang, K. C. Chen, W. Ni et al., "Big data analytics and network calculus enabling intelligent management of autonomous vehicles in a smart city," IEEE Internet Things J., vol. 6, no. 2, pp. 2021-2034, Apr. 2019.

[2] T. Shu, J. Chen, V. K. Bhargava and C. W. de Silva, "An energy-efficient dual prediction scheme using LMS filter and LSTM in wireless sensor networks for environment monitoring," IEEE Internet Things J., vol. 6, no. 4, pp. 6736-6747, Aug. 2019.

[3] P. Verma and S. K. Sood, "Fog assisted-IoT enabled patient health monitoring in smart homes," IEEE Internet Things J., vol. 5, no. 3, pp. 1789-1796, June 2018.

[4] S. Kaul, R. D. Yates, and M. Gruteser, "Real-time status: How often should one update?" in Proc. IEEE INFOCOM, pp. 2731-2735, Mar. 2012.

[5] E. Najm, R. D. Yates, and E. Soljanin, "Status update through M/G/1/1 queues with HARQ," in Proc. ISIT, pp. 131-135, Jun. 2017.

[6] R. Devassy, G. Durisi, G. C. Ferrante, O. Simeone, E. Uysal-Biyikoglu, "Reliable transmission of short packets through queues and noisy channels under latency and peak-age violation guarantees," IEEE J. Sel. Areas Commun., vol. 37, no. 4, pp. 721-734, Apr. 2018.

[7] I. Kadota, A. Sinha, E. Modiano, "Scheduling algorithms for optimizing age of information in wireless networks with throughput comstraints," IEEE/ACM Trans. Netw., vol. 27, no. 4, pp. 1369-1372, Aug. 2019.

[8] Y.-P. Hsu, E. Modiano, and L. Duan, "Age of information: Design and analysis of optimal scheduling algorithms," in Proc. IEEE ISIT, Jun. 2017.

[9] I. Kadota, A. Sinha, E. Uysal-Biyikoglu, E. Modiano, "Scheduling policies for minimizing age of information in broadcast wireless networks," IEEE/ACM Trans. Netw., vol. 26, no. 6, pp. 2637-2650, Dec. 2018.

[10] B. T. Bacinoglu and E. Uysal-Biyikoglu, "Scheduling status updates to minimize age of information with an energy harvesting sensor," in Proc. IEEE ISIT, 2017.

[11] R. D. Yates, "Lazy is timely: Status updates by an energy harvesting source," in Proc. IEEE ISIT, pp. 3008-3012, Jun. 2015.

[12] A.Arafa, S.Ulukus, "Timely updates in energy harvesting two-hop networks: offline and online policies," IEEE Trans. Wireless Commun., vol. 18, no. 8, pp. 4017-4030, Aug. 2019.

[13] X. Wu, J. Yang, and J. Wu, "Optimal status update for age of information minimization with an energy harvesting source," IEEE Trans. Green Commun. Netw., vol. 2, no. 1, pp. 193-204, Mar. 2018.

[14] I. Krikidis, "Average age of information in wireless powered sensor networks," IEEE Wireless Commun. Lett., vol. 8, no. 2, pp. 628-631, Jan. 2019.

[15] Z. Chen, K. Chi, K. Zheng, G. Dai and Q. Shao, "Minimization of transmission completion time in UAV-enabled wireless powered communication networks," IEEE Internet Things J., vol. 7, no. 2, pp. 1245-1259, Feb. 2020.

[16] S. Say, H. Inata, J. Liu, and S. Shimamoto, "Priority-based data gathering framework in UAV-assisted wireless sensor networks," IEEE Sensors J., vol. 16, no. 14, pp. 5785-5794, Jul. 2016.

[17] C. Zhan, Y. Zeng, and R. Zhang, "Energy-efficient data collection in UAV enabled wireless sensor network," IEEE Wireless Commun. Lett., vol. 7, no. 3, Jun. 2018.

[18] Y. Zeng, R. Zhang, and J. L. Teng, "Wireless communications with unmanned aerial vehicles: opportunities and challenges," IEEE Commun. Mag., vol. 54, no. 5, pp. 36-42, May 2016.

[19] Y. Liu, K. Xiong, Q. Ni, P. Fan, K. Ben Letaief, "UAV-assisted wireless powered cooperative mobile edge computing: Joint offloading, CPU control and trajectory optimization," IEEE Internet Things J., vol. 7, no. 4, pp. 2777-2790, April 2020.

[20] A. Siddiqui, L. Musavian, S. Aissa, Q. Ni, "Performance analysis of relaying systems with fixed and energy harvesting batteries," IEEE Trans. Commun., vol. 66, no. 4, pp. 1386-1398, April 2018.

[21] J. Liu, X. Wang, B. Bai, and H. Dai, "Age-optimal trajectory planning for UAV-assisted data collection," in Proc. IEEE INFOCOM, pp. 553-558, 2018.
[22] Z. Jia, X. Qin, Z. Wang, B. Liu, "Age-based path planning and data acquisition in UAV-assisted IoT networks," in Proc. IEEE ICC, Jul. 2019.

[23] P. Tong, J. Liu, X. Wang, B. Bai, H. Dai, "UAV-enabled age-optimal data collection in wireless sensor networks," in Proc. IEEE ICC, Jul. 2019.

[24] M. A. Abd-Elmagid and H. S. Dhillon, "Average peak age-ofinformation minimization in UAV-assisted IoT networks," IEEE Trans. Veh. Technol., vol. 68, no. 2, pp. 2003-2008, Feb. 2019.

[25] S. Guizani, "Low energy system proposal for UAV applications using flexible solar cells," in Proc. IEEE IWCMC. Jun. 2019.

[26] R. H. Jiang, K. Xiong, P. Y. Fan, Y. Zhang and Z. D. Zhong, "Power Minimization in SWIPT Networks With Coexisting Power-Splitting and Time-Switching Users Under Nonlinear EH Model,'” IEEE Internet Things J., vol. 6, no. 5, pp. 8853-8869, Oct. 2019.

[27] Y. Lu, K. Xiong, P. Fan, Z. Ding, Z. Zhong, and K. B. Letaief, "Global energy efficiency in secure MISO SWIPT systems with non-linear powersplitting EH model," IEEE J. Sel. Areas Commun., vol. 37, no. 1, pp. 216-232, Jan. 2019.

[28] X. Di, K. Xiong, P. Fan, H. Yang, "Simultaneous wireless information and power transfer in cooperative relay networks with rateless codes," IEEE Trans. Veh. Technol. vol. 66, no. 4, pp. 2981-2996, Apr. 2017.

[29] M. Gorlatova, A. Wallwater, G. Zussman, "Networking low-power energy harvesting devices: measurements and algorithms," IEEE Trans. Mob. Comput., vol.12, no. 9, pp. 1853-1865, Sept. 2013.

[30] H. Zheng, K. Xiong, P. Fan, Z. Zhong, K. B. Letaief, "Fog-assisted multi-user SWIPT networks: local computing or offloading?" IEEE Internet Things J., vol. 6, no. 3, pp. 5246-5264, Jun. 2019.

[31] K. Xiong, P. Fan, C. Zhang, K.B. Letaief, "Wireless information and energy transfer for two-hop non-regenerative MIMO-OFDM relay networks," IEEE J. Sel. Areas Commun., vol. 33, no. 8, pp. 1595-1611, Aug. 2015.

[32] L. Xie, J. Xu and R. Zhang, "Throughput maximization for UAV-enabled wireless powered communication networks," IEEE Internet Things J., vol. 6, no. 2, pp. 1690-1703, Apr. 2019.

[33] J. Xu, Y. Zeng and R. Zhang, "UAV-enabled wireless power transfer: trajectory design and energy optimization," IEEE Trans. Wireless Commun., vol. 17, no. 8, pp. 5092-5106, Aug. 2018.

[34] K. Li, W. Ni, E. Tovar and A. Jamalipour, "On-board deep Q-network for UAV-assisted online power transfer and data collection," IEEE Trans. Veh. Technol., vol. 68, no. 12, pp. 12215-12226, Dec. 2019.

[35] L. Yang, J. Chen, M. O. Hasna, H. C. Yang, "Outage performance of UAV-assisted relaying systems with RF energy harvesting," IEEE Commun. Lett., vol. 22, no. 12, pp. 2471-2474, Dec. 2018.

[36] H. Wang, J. Wang, G. Ding, L. Wang, T. A. Tsiftsis, and P. K. Sharma, "Resource allocation for energy harvesting-powered D2D communication underlaying UAV-assisted networks," IEEE Trans. Green Commun. Netw., vol. 2, no. 1, pp. 14-24, Mar. 2018.

[37] Y. Lu, K. Xiong, P. Fan, Z. Zhong, and K. B. Letaief, "Robust transmit beamforming with artificial redundant signals for secure SWIPT system under non-linear EH model," IEEE Trans. Wireless Commun., vol. 17, no. 4, pp. 2218-2232, Apr. 2018.

[38] S. Boyd and L. Vandenberghe, Convex Optimization. Cambridge, U.K.: Cambridge Univ. Press, 2004.

[39] I. Chatzigeorgiou, "Bounds on the Lambert function and their application to the outage analysis of user cooperation," IEEE Commun. Lett., vol. 17, no. 8, pp. 1505-1508, Aug. 2013.

[40] B. Clerckx and E. Bayguzina, "Waveform design for wireless power transfer," IEEE Trans. Signal Process., vol. 64, no. 23, pp. 6313-6328, Dec. 2016.

[41] Y. Lu, K. Xiong, P. Fan, Z. Ding, Z. Zhong, and K. B. Letaief, "Global energy efficiency in secure MISO SWIPT systems with non-linear powersplitting EH model," IEEE J. Sel. Areas Commun., vol. 37, no. 1, pp. 216-232, Jan. 2019.

[42] N. Nguyen, B. Liu, "The mobile sensor deployment problem and the target coverage problem in mobile wireless sensor networks are NPHard," IEEE Syst. J., vol. 13, no. 2, pp. 1312-1315, Jun. 2019.

[43] T. Bektas, "The multiple traveling salesman problem: an overview of formulations and solution procedures," Omega, vol. 34, no. 3, pp. 209$219,2006$.

[44] K. Liu, M. Zhang, "Path planning based on simulated annealing ant colony algorithm," in Proc. IEEE ISCID, pp. 2473-3547, Dec. 2016.

[45] W. Zhao, X. Cai and Y. Lan, "A new ant colony algorithm for solving traveling salesman problem," in Proc. IEEE ICCSEE, pp. 530-533, Apr. 2012,

[46] D. Yang, Q. Wu, Y. Zeng and R. Zhang, "Energy tradeoff in ground-toUAV communication via trajectory design," IEEE Trans. Veh. Technol., vol. 67, no. 7, pp. 6721-6726, Jul. 2018. 
[47] E. Boshkovska, D. W. K. Ng, N. Zlatanov, and R. Schober, "Practical non-linear energy harvesting model and resource allocation for SWIPT systems," IEEE Commun. Lett., vol. 19, no. 12, pp. 2082-2085, Dec. 2015.

[48] E. Boshkovska, D. W. K. Ng, N. Zlatanov, A. Koelpin, and R. Schober "Robust resource allocation for MIMO wireless powered communication networks based on a non-linear EH model," IEEE Trans. Commun., vol. 65, no. 5, pp. 1984-1999, May 2017.

[49] C. R. Valenta and G. D. Durgin, "Harvesting wireless power: survey of energy-harvester conversion efficiency in far-field, wireless power transfer systems," IEEE Microw. Mag., vol. 15, no. 4, pp. 108-120, Jun. 2014.

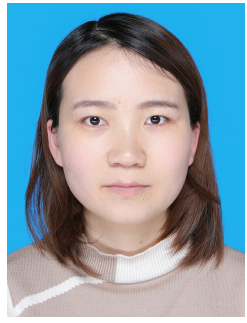

Huimin Hu received the B.S. degree from School of Electronic and Information Engineering, Lanzhou Jiaotong University, Lanzhou, China, in 2017. She is currently pursuing the Ph.D. degree with the School of Computer and Information Technology, Beijing Jiaotong University, Beijing, China. Her current research interests include Age of Information, energy harvesting in wireless communication networks, wireless sensor networks.

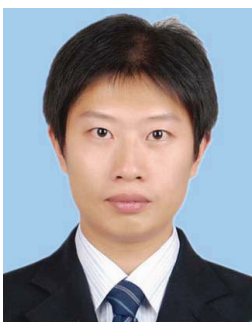

Ke Xiong (M'14) received the B.S. and Ph.D. degrees from Beijing Jiaotong University (BJTU), Beijing, China, in 2004 and 2010, respectively. From April 2010 to February 2013, he was a Postdoctoral Research Fellow with the Department of Electronics Engineering, Tsinghua University, Beijing. Since March 2013, he has been a Lecturer and an associate professor of BJTU. From Sept. 2015 to Sept. 2016, he was a visiting scholar at University of Maryland, College Park. He is currently a full professor with the School of Computer and Information Technology, BJTU. His research interests include wireless cooperative networks, wireless powered networks, and network information theory.

He has published more than 100 academic papers in referred journals and conferences. He is a member of China Computer Federation (CCF) and also a senior member of the Chinese Institute of Electronics (CIE). Dr. Xiong serves as an Associate Editor-in Chief of the Chinese journal New Industrialization Strategy, and the editor of Computer Engineering \& Software. In 2017, he served as the leading editor of the Special issue "Recent Advances in Wireless Powered Communication Networks" for EURASIP Journal on Wireless Communications and Networking. Currently, he also serves as a reviewer more than 15 international journals including IEEE Transactions on Signal Processing, IEEE Transactions on Wireless Communications, IEEE Transactions on Communications, IEEE Transactions on Vehicular Technology, IEEE Communication Letters, IEEE Signal Processing Letters and IEEE Wireless Communication Letters. He also served as a Session Chair for IEEE GLOBECOM2012, IET ICWMMN2013, IEEE ICC2013, ACM MOMM2014 and the Publicity and Publication Chair for IEEE HMWC2014, as well as the TPC co-chair of IET ICWMMN2017 and IET ICWMMN2019.

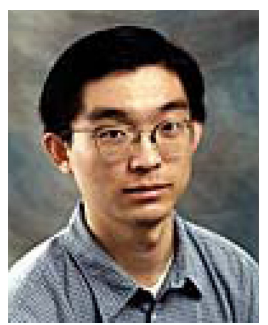

Gang Qu received the Ph.D. degree in computer science from the University of California, Los Angeles. He is currently a Professor with the Department of Electrical and Computer Engineering and the Institute for Systems Research, University of Maryland at College Park. He is also a member of the Maryland Cybersecurity Center and the Maryland Energy Research Center. He is the Director of the Maryland Embedded Systems and Hardware Security Laboratory and the Wireless Sensors Laboratory. His primary research interests are in the area of embedded systems and VLSI CAD with a focus on low power system design and hardware related security and trust. $\mathrm{He}$ is an enthusiastic teacher, and has taught and co-taught various security courses, including VLSI design intellectual property protection, cybersecurity for smart grid, reverse engineering and hardware security lab, and a popular MOOC on hardware security through courser.

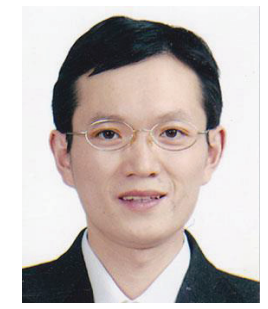

Qiang Ni (M'04-SM'08) received the B.Sc., M.Sc., and Ph.D. degrees from the Huazhong University of Science and Technology, China, all in engineering. $\mathrm{He}$ is currently a Professor and the Head of the Communication Systems Group, School of Computing and Communications, Lancaster University, Lancaster, U.K. His research interests include the area of future generation communications and networking, including green communications and networking, millimeter-wave wireless communications, cognitive radio network systems, non-orthogonal multiple access (NOMA), heterogeneous networks, 5G and 6G, SDN, cloud networks, energy harvesting, wireless information and power transfer, IoTs, cyber physical systems, AI and machine learning, big data analytics, and vehicular networks. He has authored or co-authored over 200 papers in these areas. He was an IEEE 802.11 Wireless Standard Working Group Voting Member and a contributor to the IEEE Wireless Standards.

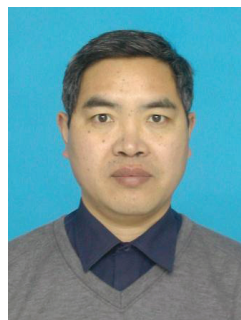

Pingyi Fan (M'03-SM'09) received the B.S and M.S. degrees from the Department of Mathematics of Hebei University in 1985 and Nankai University in 1990 , respectively, received his Ph.D degree from the Department of Electronic Engineering, Tsinghua University, Beijing, China in 1994. He is a professor of department of EE of Tsinghua University currently. From Aug. 1997 to March. 1998, he visited Hong Kong University of Science and Technology as Research Associate. From May. 1998 to Oct. 1999, he visited University of Delaware, USA, as research fellow. In March. 2005, he visited NICT of Japan as visiting Professor. From June. 2005 to May 2014, he visited Hong Kong University of Science and Technology for many times and From July 2011 to Sept. 2011, he is a visiting professor of Institute of Network Coding, Chinese University of Hong Kong.

Dr. Fan is a senior member of IEEE and an oversea member of IEICE. He has attended to organize many international conferences including as General co-Chair of IEEE VTS HMWC2014, TPC co-Chair of IEEE International Conference on Wireless Communications, Networking and Information Security (WCNIS 2010) and TPC member of IEEE ICC, Globecom, WCNC, VTC, Inforcom etc. He has served as an editor of IEEE Transactions on Wireless Communications, Inderscience International Journal of Ad Hoc and Ubiquitous Computing and Wiley Journal of Wireless Communication and Mobile Computing. He is also a reviewer of more than 30 international Journals including 20 IEEE Journals and 8 EURASIP Journals. He has received some academic awards, including the IEEE Globecom'14 Best Paper Award, IEEE WCNC'08 Best Paper Award, ACM IWCMC'10 Best Paper Award and IEEE ComSoc Excellent Editor Award for IEEE Transactions on Wireless Communications in 2009. His main research interests include B5G technology in wireless communications such as MIMO, OFDMA, etc., Network Coding, Network Information Theory and Big Data Analysis etc. 


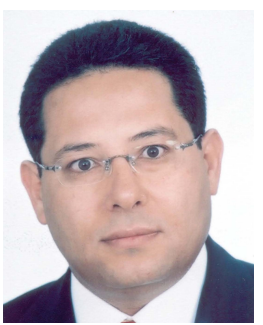

Khaled Ben Letaief (S'85-M'86-SM'97-F'03) received his Ph.D. from Purdue University, USA. He is Chair Professor and Provost of HBKU, a newly established research-intensive university in Qatar. $\mathrm{He}$ has served as HKUST Dean of Engineering from 2009 to 2015. Under his leadership, HKUST School of Engineering has not only transformed its education and scope and produced very high caliber scholarship, it has also actively pursued knowledge transfer and societal engagement in broad contexts. It has also dazzled in international rankings. $\mathrm{He}$ is currently also with the Peng Cheng Laboratory, Shenzhen, China.

Dr. Letaief is a world-renowned leader in wireless communications and networks. In these areas, he has over 620 journal and conference papers and given invited keynote talks as well as courses all over the world. $\mathrm{He}$ has made 6 major contributions to IEEE Standards along with 15 patents. He served in many leadership positions, including Dean of Engineering at HKUST, Provost at HBKU, Director of the Hong Kong Telecom Institute of Information Technology, Vice-President of IEEE Communications Society, and Founding Editor-in-Chief of the well-regarded IEEE Transactions on Wireless Communications. He also served as the President of the IEEE Communications Society (2018-19), the world's leading organization for communications professionals with headquarter in New York City and members in 162 countries.

$\mathrm{He}$ is the recipient of many prestigious awards, including the 2019 Distinguished Research Excellence Award by HKUST School of Engineering; 2019 IEEE Communications Society and Information Theory Society Joint Paper Award; 2018 IEEE Signal Processing Society Young Author Best Paper Award; 2016 IEEE Marconi Prize Award in Wireless Communications; and Purdue University Outstanding Electrical and Computer Engineer Award. $\mathrm{He}$ has also received many distinguished awards in the field of teaching excellence, including the Michael G. Gale Medal for Distinguished Teaching (Highest university-wide teaching award at HKUST). 